\title{
Central African biomes and forest succession stages derived from modern pollen data and plant functional types
}

\author{
J. Lebamba ${ }^{1}$, A. Ngomanda ${ }^{2}$, A. Vincens ${ }^{3}$, D. Jolly ${ }^{1, \dagger}$, C. Favier ${ }^{1}$, H. Elenga ${ }^{4}$, and I. Bentaleb ${ }^{1,5}$ \\ ${ }^{1}$ ISE-M, UMR 5554 CNRS/Université Montpellier II, Place Eugène Bataillon, cc61, 34095 Montpellier cedex 5, France \\ ${ }^{2}$ Institut de Recherche en Ecologie Tropicale (IRET/CENAREST), BP 13354, Libreville, Gabon \\ ${ }^{3}$ CEREGE, CNRS/Université Aix-Marseille/IRD/CdF, BP 80, 13545 Aix-en-Provence cedex 04, France \\ ${ }^{4}$ Faculté des Sciences, Université Marien Ngouabi, BP 69, Brazzaville, Congo \\ ${ }^{5}$ LSCE, UMR CNRS/CEA/UVSQ, 12 Avenue de la Terrasse, 91198 Gif-sur-Yvette cedex, France \\ $\dagger$ deceased
}

Received: 23 October 2008 - Published in Clim. Past Discuss.: 15 January 2009

Revised: 20 May 2009 - Accepted: 17 June 2009 - Published: 29 July 2009

\begin{abstract}
New detailed vegetation reconstructions are proposed in Atlantic Central Africa from a modern pollen data set derived from 199 sites (Cameroon, Gabon and Congo) including 131 new sites. In this study, the concept of plant functional classification is improved with new and more detailed plant functional types (PFTs) and new aggregations of pollen taxa. Using the biomisation method, we reconstructed (1) modern potential biomes and (2) potential succession stages of forest regeneration, a new approach in Atlantic Central African vegetation dynamics and ecosystem functioning reconstruction. When compared to local vegetation, potential biomes are correctly reconstructed $(97.5 \%$ of the sites) and tropical rain forest (TRFO biome) is well identified from tropical seasonal forest (TSFO biome). When the potential biomes are superimposed on the White's vegetation map, only $76.4 \%$ of the sites are correctly reconstructed. But using botanical data, correspondence and cluster analyses, the 43 sites from Congo (Mayombe) evidence more affinities with those of central Gabon and so they can also be considered as correctly reconstructed as TRFO biome and White's map should be revised. In terms of potential succession stages of forest regeneration, the mature forest (TMFO) is well differentiated from the secondary forest (TSFE), but inside this latter group, the young and the pioneer stages are not clearly identified due probably to their low sampling representation. Moreover, linked to their progressive and mosaic character, the boundaries between two forest biomes or two forest stages are not clearly detected and need also a more intensive sampling in such transitions.
\end{abstract}

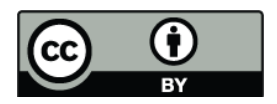

Correspondence to: J. Lebamba (judi@isem.univ-montp2.fr)

\section{Introduction}

Plant functional classifications were first proposed by a core project of IGBP, "Global Change and Terrestrial Ecosystems", in the early-mid 1990's as a tool to model vegetation dynamics and ecosystem functioning in response to climate and $\mathrm{CO}_{2}$. Such classifications appeared as an ecological alternative to traditional taxonomic entities for the simplification of floristic complexity in global vegetation models (e.g. Prentice et al., 1992; Woodward and Cramer, 1996; Haxeltine and Prentice, 1996; Cramer, 1997; Leemans, 1997; Smith et al., 1997) and for mapping vegetation patterns at key periods in the past (Prentice and Webb, 1998; Prentice et al., 2000). Since then, extensive research has been carried out to identify plant functional types (PFTs) which are sets of plant species exhibiting similar responses to environmental conditions and grouped on the basis of structural and functional characters (e.g. Noble and Gitay, 1996; Diaz and Cabido, 1997; Diaz Barradas et al., 1999). These PFTs are characterised by a set of common biological attributes correlated with their behaviour (Gitay and Noble, 1997; Lavorel et al., 1997; Lavorel and Garnier, 2002; Lavorel et al., 2007).

In Africa, the notion of plant functional types by the means of pollen data was developed for the first time by Jolly et al. (1998a, b), then by Elenga et al. (2000a), Peyron et al. (2000, 2006), Ngomanda (2005) and Vincens et al. (2006) to reconstruct biomes and climate at key periods (Present, $6000 \mathrm{BP}$ and $18000 \mathrm{BP}$ ) or along quaternary pollen sequences. Skarpe (1996), Jolly and Haxeltine (1997) and Hély et al. (2006) have also used such a classification to test the sensitivity of African biomes to changes in climatic parameters (e.g. the precipitation regime or the temperature). According to the high species diversity of the African flora,

Published by Copernicus Publications on behalf of the European Geosciences Union. 


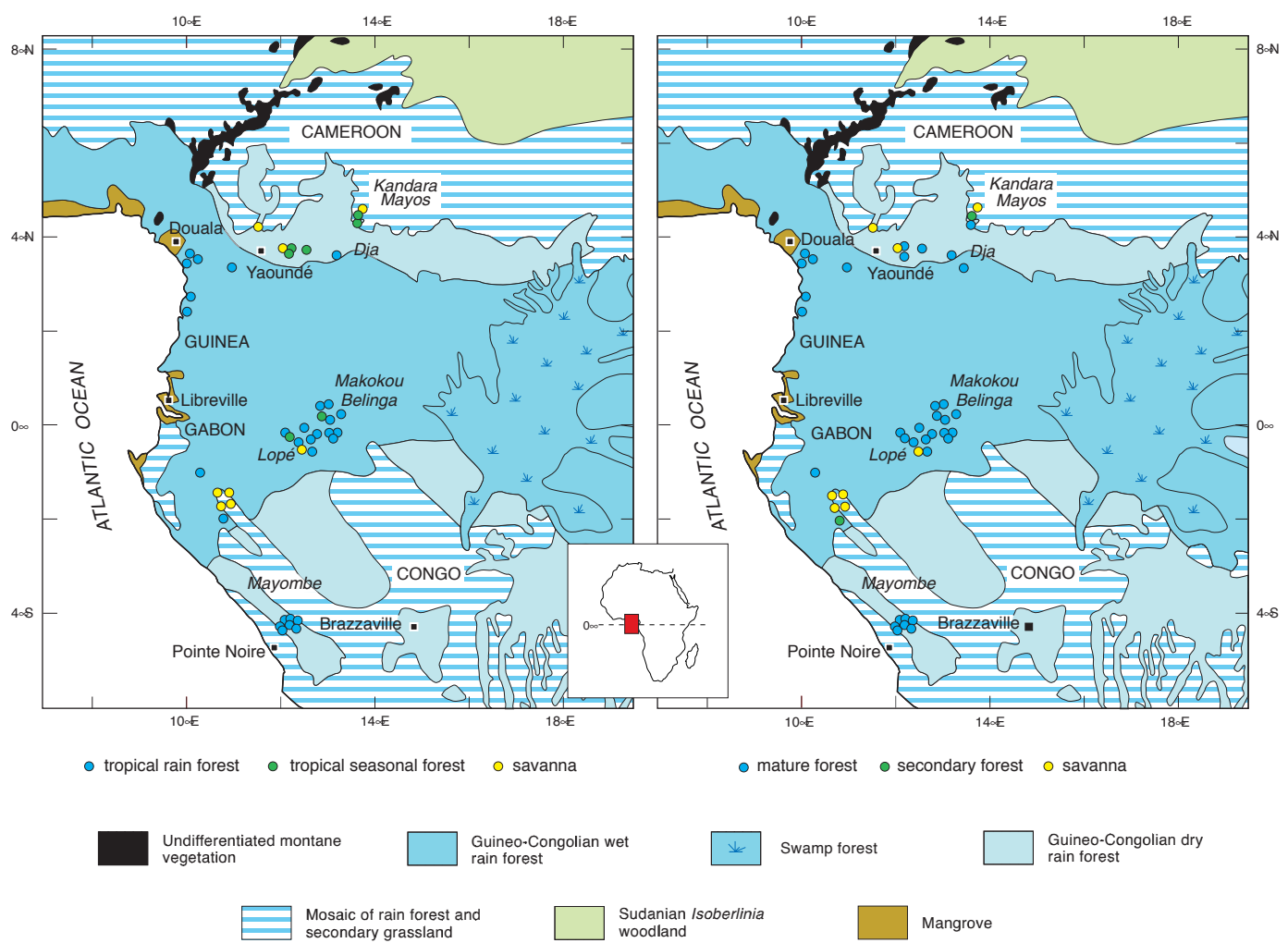

Fig. 1. Location of modern pollen samples in Central Africa according to White's vegetation map. (a) Reconstructed potential biomes (TRFO: Tropical rain forest; TSFO: Tropical seasonal forest; SAVA: Savanna), (b) Reconstructed succession stages of forest regeneration.

plant functional classifications have proved to be a good tool for understanding the present, past and future functioning of the African ecosystems.

The aim of this paper is to apply this concept of classification on a large modern pollen data set (199 pollen assemblages, 272 taxa) from Atlantic Central Africa. In this region the main ecosystems, particularly the forest ones, were not always previously accurately identified by their pollen rain due to their low sampling representation, providing difficult a comparison of the data with vegetation models. Plant functional classifications, associated with the biomisation method (Prentice et al., 1996), were used following two ways: (1) the reconstruction of modern potential biomes and (2) the reconstruction of potential succession stages of forest regeneration, a new approach in Atlantic Central African vegetation dynamics and ecosystem functioning reconstruction. New PFTs and assignment of pollen taxa in these plant functional types are proposed.

\section{Botanical environmental setting}

The study area, covering the southern Cameroon, the Gabon and the southern Congo, is located between latitude $5^{\circ} \mathrm{N}$ and $5^{\circ} \mathrm{S}$ and between longitude $10^{\circ}$ and $15^{\circ} \mathrm{E}$ (Fig. 1; Table 1).
It is mainly occupied by lowlands, with an average altitude of $400 \mathrm{~m}$

This area is floristically located in the Guineo-Congolian centre of endemism (White, 1983). In this region three main types of vegetation occurring on well-drained soils are differentiated by this author.

\subsection{The wetter types of Guineo-Congolian rain forest}

These types of forest includes: (i) The coastal evergreen rain forest characterized by the presence of Sacoglottis gabonensis and Lophira alata in Cameroon (Letouzey, 1957; Tchouto Mbatchou, 2004), this latter tree being replaced by Okoumea klaineana in Gabon (De Saint Aubin, 1963; Caballé, 1978) and Congo (Hecketsweiler and Mokoko Ikonga, 1991; Doumenge, 1992). This forest type is called "Atlantic littoral forest" by Letouzey (1968, 1985). Inland, on the small hills and low mountains, this evergreen rain forest is replaced by an evergreen rain forest very rich in Caesalpiniaceae ("Biafran forest", Letouzey, 1968, 1985). (ii) The mixed moist semi-evergreen rain forest ("Congolian forest", Letouzey, 1968, 1985), well developed in southeastern Cameroon (Letouzey, 1968, 1985) and the eastern half Gabon (Nicolas, 1977) is characterized by a mixture of evergreen and some semi-deciduous species becoming more 
Table 1. Location of modern pollen samples in Atlantic Central Africa, derived vegetation and reconstructed biomes and succession stages (column A, authors of the data: (a) Vincens, unpublished; (b) Lebamba et al., 2009; (c) Vincens et al., 2000; (d) Jolly et al., 1996; (e) Ngomanda, unpublished; (f) Elenga et al., 2000b); (Reconstructed biomes: TRFO (Tropical Rain Forest), TSFO (Tropical Seasonal Forest) and SAVA (savanna); Reconstructed stages: TMFO (Tropical Mature Forest), TOSF (Tropical Old Secondary Forest), TYSF (Tropical young Secondary Forest) and SAVA (Savanna). (in bold italics, not correctly reconstructed potential biomes and stages compared to local vegetation).

\begin{tabular}{|c|c|c|c|c|c|c|c|c|}
\hline Samples & Location & Country & Lat. & Long. & Alt (m) & Local observed vegetation & Reconstructed biomes & Reconstructed stages \\
\hline 1C83 (b) & Ndokoua & Cameroon & 4.38 & 11.72 & 516 & Savanna & SAV & SAVA \\
\hline ka1620 (a) & Kandara Nord & Cameroon & 4.33 & 13.72 & 640 & mature semi-deciduous forest & TSFO & TOSF \\
\hline ka1600 (a) & Kandara Nord & Cameroon & 4.33 & 13.72 & 640 & mature semi-deciduous forest & TSFO & TMFO \\
\hline ka1580 (a) & Kandara Nord & Cameroon & 4.33 & 13.72 & 640 & mature semi-deciduous forest & TSFO & TMFO \\
\hline ka1560 (a) & Kandara Nord & Cameroon & 4.33 & 13.72 & 640 & mature semi-deciduous forest & TSFO & TOSF \\
\hline ka1540 (a) & Kandara Nord & Cameroon & 4.33 & 13.72 & 640 & old secondary semi-deciduous forest & TSFO & TMFO \\
\hline ka1520 (a) & Kandara Nord & Cameroon & 4.33 & 13.72 & 640 & old secondary semi-deciduous forest & TSFO & TOSF \\
\hline ka1500 (a) & Kandara Nord & Cameroon & 4.33 & 13.72 & 640 & old secondary semi-deciduous forest & TSFO & TOSF \\
\hline ka1480 (a) & Kandara Nord & Cameroon & 4.33 & 13.72 & 640 & old secondary semi-deciduous forest & TSFO & TOSF \\
\hline ka1460 (a) & Kandara Nord & Cameroon & 4.33 & 13.72 & 640 & old secondary semi-deciduous forest & TSFO & TOSF \\
\hline ka1440 (a) & Kandara Nord & Cameroon & 4.33 & 13.72 & 640 & young secondary semi-deciduous forest & TSFO & TMFO \\
\hline ka1420 (a) & Kandara Nord & Cameroon & 4.33 & 13.72 & 640 & young secondary semi-deciduous forest & TSFO & TOSF \\
\hline ka1400 (a) & Kandara Nord & Cameroon & 4.33 & 13.72 & 640 & young secondary semi-deciduous forest & TSFO & TOSF \\
\hline ka1380 (a) & Kandara Nord & Cameroon & 4.33 & 13.72 & 640 & young secondary semi-deciduous forest & TSFO & TOSF \\
\hline ka1360 (a) & Kandara Nord & Cameroon & 4.33 & 13.72 & 640 & young secondary semi-deciduous forest & TSFO & TOSF \\
\hline ka1300 (a) & Kandara Nord & Cameroon & 4.33 & 13.72 & 640 & savanna & TSFO & TOSF \\
\hline ka1280 (a) & Kandara Nord & Cameroon & 4.33 & 13.72 & 640 & savanna & SAV & SAVA \\
\hline ka1260 (a) & Kandara Nord & Cameroon & 4.33 & 13.72 & 640 & savanna & SAV & SAVA \\
\hline ka1240 (a) & Kandara Nord & Cameroon & 4.33 & 13.72 & 640 & forest regrowth & TSFO & $T M F O$ \\
\hline ka1220 (a) & Kandara Nord & Cameroon & 4.33 & 13.72 & 640 & forest regrowth & $S A V$ & SAVA \\
\hline ka1200 (a) & Kandara Nord & Cameroon & 4.33 & 13.72 & 640 & forest regrowth & TSFO & SAVA \\
\hline ka735 (c) & Kandara Sud & Cameroon & 4.33 & 13.72 & 640 & savanna & SAV & SAVA \\
\hline ka705 (c) & Kandara Sud & Cameroon & 4.33 & 13.72 & 640 & savanna & SAV & SAVA \\
\hline ka675 (c) & Kandara Sud & Cameroon & 4.33 & 13.72 & 640 & savanna & SAV & SAVA \\
\hline ka645 (c) & Kandara Sud & Cameroon & 4.33 & 13.72 & 640 & savanna & SAV & SAVA \\
\hline ka615 (c) & Kandara Sud & Cameroon & 4.33 & 13.72 & 640 & savanna & SAV & SAVA \\
\hline ka585 (c) & Kandara Sud & Cameroon & 4.33 & 13.72 & 640 & savanna & SAV & SAVA \\
\hline ka555 (c) & Kandara Sud & Cameroon & 4.33 & 13.72 & 640 & savanna & SAV & SAVA \\
\hline ka525 (c) & Kandara Sud & Cameroon & 4.33 & 13.72 & 640 & savanna & SAV & SAVA \\
\hline ka502 (c) & Kandara Sud & Cameroon & 4.33 & 13.72 & 640 & forest regrowth & TSFO & TYSF \\
\hline ka480 (c) & Kandara Sud & Cameroon & 4.33 & 13.72 & 640 & forest regrowth & TSFO & TOSF \\
\hline ka450 (c) & Kandara Sud & Cameroon & 4.33 & 13.72 & 640 & young secondary semi-deciduous forest & TSFO & TOSF \\
\hline ka420 (c) & Kandara Sud & Cameroon & 4.33 & 13.72 & 640 & young secondary semi-deciduous forest & TSFO & TOSF \\
\hline ka390 (c) & Kandara Sud & Cameroon & 4.33 & 13.72 & 640 & young secondary semi-deciduous forest & TSFO & TOSF \\
\hline ka360 (c) & Kandara Sud & Cameroon & 4.33 & 13.72 & 640 & young secondary semi-deciduous forest & TSFO & TOSF \\
\hline ka330 (c) & Kandara Sud & Cameroon & 4.33 & 13.72 & 640 & young secondary semi-deciduous forest & TSFO & TOSF \\
\hline ka300 (c) & Kandara Sud & Cameroon & 4.33 & 13.72 & 640 & old secondary semi-deciduous forest & TSFO & TOSF \\
\hline ka270 (c) & Kandara Sud & Cameroon & 4.33 & 13.72 & 640 & old secondary semi-deciduous forest & TSFO & $T M F O$ \\
\hline ka240 (c) & Kandara Sud & Cameroon & 4.33 & 13.72 & 640 & old secondary semi-deciduous forest & TSFO & TOSF \\
\hline ka210 (c) & Kandara Sud & Cameroon & 4.33 & 13.72 & 640 & mature semi-deciduous forest & TSFO & TOSF \\
\hline ka180 (c) & Kandara Sud & Cameroon & 4.33 & 13.72 & 640 & mature semi-deciduous forest & TSFO & TOSF \\
\hline ka150 (c) & Kandara Sud & Cameroon & 4.33 & 13.72 & 640 & mature semi-deciduous forest & TSFO & TMFO \\
\hline
\end{tabular}


Table 1. Continued.

\begin{tabular}{|c|c|c|c|c|c|c|c|c|}
\hline Samples & Location & Country & Lat. & Long. & Alt (m) & Local observed vegetation & $\begin{array}{l}\text { Reconstructed } \\
\text { biomes }\end{array}$ & $\begin{array}{l}\text { Reconstructed } \\
\text { stages }\end{array}$ \\
\hline ka120 (c) & Kandara Sud & Cameroon & 4.33 & 13.72 & 640 & mature semi-deciduous forest & TSFO & TMFO \\
\hline $\mathrm{ka90}(\mathrm{c})$ & Kandara Sud & Cameroon & 4.33 & 13.72 & 640 & mature semi-deciduous forest & TSFO & TMFO \\
\hline ka60 (c) & Kandara Sud & Cameroon & 4.33 & 13.72 & 640 & mature semi-deciduous forest & TSFO & TMFO \\
\hline ka40 (c) & Kandara Sud & Cameroon & 4.33 & 13.72 & 640 & mature semi-deciduous forest & TSFO & TMFO \\
\hline may1 (a) & Mayos & Cameroon & 4.32 & 13.57 & 600 & old secondary semi-deciduous forest & TSFO & $T M F O$ \\
\hline may2 (a) & Mayos & Cameroon & 4.32 & 13.57 & 600 & old secondary semi-deciduous forest & TSFO & TMFO \\
\hline may3 (a) & Mayos & Cameroon & 4.32 & 13.57 & 600 & mature semi-deciduous forest & TSFO & TMFO \\
\hline C58 (b) & Nebodo & Cameroon & 4.01 & 12.64 & 662 & mature semi-deciduous forest & TSFO & TMFO \\
\hline C84 (b) & Nyek & Cameroon & 4.00 & 12.22 & 693 & savanna & SAV & SAVA \\
\hline C60 (b) & Biba & Cameroon & 3.88 & 12.24 & 667 & mature semi-deciduous forest & TSFO & TMFO \\
\hline C61 (b) & Biba & Cameroon & 3.86 & 12.24 & 671 & mature semi-deciduous forest & TSFO & TMFO \\
\hline C62 (b) & Biba & Cameroon & 3.85 & 12.24 & 643 & mature semi-deciduous forest & TSFO & TMFO \\
\hline C63 (b) & Biba & Cameroon & 3.85 & 12.24 & 687 & mature semi-deciduous forest & TSFO & TMFO \\
\hline C75 (b) & Cyrie (Dja) & Cameroon & 3.82 & 13.31 & 668 & mature rain forest/semi-deciduous forest transition & TRFO & TMFO \\
\hline C76 (b) & Cyrie (Dja) & Cameroon & 3.82 & 13.31 & 678 & mature rain forest/semi-deciduous forest transition & TRFO & TMFO \\
\hline $\mathrm{C} 77$ (b) & Cyrie (Dja) & Cameroon & 3.82 & 13.31 & 684 & mature rain forest/semi-deciduous forest transition & TRFO & TMFO \\
\hline C78 (b) & Cyrie (Dja) & Cameroon & 3.82 & 13.31 & 681 & mature rain forest/semi-deciduous forest transition & TSFO & TMFO \\
\hline C66 (b) & Tissongo & Cameroon & 3.58 & 11.00 & 26 & mature rain forest & TRFO & TMFO \\
\hline C67 (b) & Tissongo & Cameroon & 3.58 & 11.00 & 14 & mature rain forest & TRFO & TMFO \\
\hline C68 (b) & Tissongo & Cameroon & 3.58 & 11.00 & 11 & mature rain forest & TRFO & TMFO \\
\hline C69 (b) & Nsah & Cameroon & 3.56 & 11.00 & 17 & mature rain forest & TRFO & TMFO \\
\hline C70 (b) & Nsah & Cameroon & 3.54 & 11.00 & 19 & mature rain forest & TRFO & TMFO \\
\hline C72 (b) & Nsah & Cameroon & 3.54 & 11.00 & 15 & mature rain forest & TRFO & TMFO \\
\hline C71 (b) & Nsah & Cameroon & 3.54 & 11.00 & 19 & mature rain forest & $T S F O$ & TMFO \\
\hline C82 (b) & Nkoul (Nja) & Cameroon & 3.36 & 13.52 & 697 & mature rain forest/semi-deciduous forest transition & TRFO & TMFO \\
\hline $\mathrm{C} 80(\mathrm{~b})$ & Nkoul (Nja) & Cameroon & 3.36 & 13.52 & 697 & mature rain forest/semi-deciduous forest transition & TSFO & TMFO \\
\hline C81 (b) & Nkoul (Nja) & Cameroon & 3.36 & 13.52 & 699 & young secondary rain forest/semi-deciduous forest transition & TSFO & $T M F O$ \\
\hline C73 (b) & Nkoul (Nja) & Cameroon & 3.35 & 13.53 & 710 & mature rain forest/semi-deciduous forest transition & TRFO & TMFO \\
\hline C74 (b) & Nkoul (Nja) & Cameroon & 3.35 & 13.53 & 716 & mature rain forest/semi-deciduous forest transition & TSFO & TMFO \\
\hline eb1 (a) & Eboundja & Cameroon & 2.82 & 9.90 & 10 & mature rain forest & TRFO & TMFO \\
\hline eb2 (a) & Boussibelika & Cameroon & 2.73 & 9.87 & 20 & mature rain forest & TRFO & TMFO \\
\hline GA2B (d) & Belinga & Gabon & 1.12 & 13.12 & 925 & mature rain forest & TRFO & TMFO \\
\hline GA1M (d) & Makokou & Gabon & 0.65 & 12.71 & 470 & mature rain forest & TRFO & TMFO \\
\hline GA2M (d) & Makokou & Gabon & 0.65 & 12.71 & 470 & mature rain forest & TRFO & TMFO \\
\hline GA9M (d) & Makokou & Gabon & 0.65 & 12.71 & 470 & mature rain forest & TRFO & TMFO \\
\hline G10M (d) & Makokou & Gabon & 0.65 & 12.71 & 470 & mature rain forest & TRFO & TMFO \\
\hline G12M (d) & Makokou & Gabon & 0.65 & 12.71 & 470 & mature rain forest & TRFO & TMFO \\
\hline G13M (d) & Makokou & Gabon & 0.65 & 12.71 & 470 & mature rain forest & TRFO & TMFO \\
\hline G14M (d) & Makokou & Gabon & 0.65 & 12.71 & 470 & mature rain forest & TRFO & TMFO \\
\hline G16M (d) & Makokou & Gabon & 0.65 & 12.71 & 470 & mature rain forest & TRFO & TMFO \\
\hline G17M (d) & Makokou & Gabon & 0.65 & 12.71 & 470 & mature rain forest & TRFO & TMFO \\
\hline G18M (d) & Makokou & Gabon & 0.65 & 12.71 & 470 & mature rain forest & TRFO & TMFO \\
\hline G19M (d) & Makokou & Gabon & 0.65 & 12.71 & 470 & mature rain forest & TRFO & TMFO \\
\hline G20M (d) & Makokou & Gabon & 0.65 & 12.71 & 470 & mature rain forest & TRFO & TMFO \\
\hline $\mathrm{G} 21 \mathrm{M}(\mathrm{d})$ & Makokou & Gabon & 0.65 & 12.71 & 470 & mature rain forest & TRFO & TMFO \\
\hline G25M (d) & Makokou & Gabon & 0.65 & 12.71 & 470 & mature rain forest & TRFO & TMFO \\
\hline
\end{tabular}


Table 1. Continued.

\begin{tabular}{|c|c|c|c|c|c|c|c|c|}
\hline Samples & Location & Country & Lat. & Long. & Alt (m) & Local observed vegetation & $\begin{array}{l}\text { Reconstructed } \\
\text { biomes }\end{array}$ & $\begin{array}{l}\text { Reconstructed } \\
\text { stages }\end{array}$ \\
\hline G26M (d) & Makokou & Gabon & 0.65 & 12.71 & 470 & mature rain forest & TRFO & TMFO \\
\hline G50 (b) & Ntsiete & Gabon & 0.48 & 13.08 & 501 & mature rain forest & TRFO & TMFO \\
\hline G20 (b) & Ntsiete & Gabon & 0.40 & 13.04 & 528 & mature rain forest & TRFO & TMFO \\
\hline G22 (b) & Makokou & Gabon & 0.37 & 12.21 & 442 & mature rain forest & TRFO & TMFO \\
\hline G51(b) & Ntsiete & Gabon & 0.37 & 13.12 & 534 & mature rain forest & TSFO & TMFO \\
\hline G23 (b) & Ovan & Gabon & 0.33 & 12.07 & 413 & mature rain forest & TRFO & TMFO \\
\hline G52 (b) & Ntsiete & Gabon & 0.33 & 13.18 & 554 & mature rain forest & TRFO & TMFO \\
\hline G16 (b) & Ivindo (camp) & Gabon & 0.18 & 12.54 & 327 & mature rain forest & TRFO & TMFO \\
\hline G48 (b) & Djidji & Gabon & 0.02 & 12.40 & 420 & mature rain forest & TRFO & TMFO \\
\hline G47 (b) & Djidji & Gabon & 0.01 & 12.44 & 500 & mature rain forest & TRFO & TMFO \\
\hline G19 (b) & Djjidji & Gabon & 0.01 & 12.44 & 540 & mature rain forest & TRFO & TMFO \\
\hline G46 (b) & Djidji & Gabon & 0.01 & 12.44 & 540 & mature rain forest & TSFO & TMFO \\
\hline G49 (b) & Djidji & Gabon & -0.02 & 12.35 & 424 & mature rain forest & TRFO & TMFO \\
\hline G45 (b) & Ivindo National Park & Gabon & -0.16 & 12.51 & 547 & mature rain forest & TRFO & TMFO \\
\hline G12 (b) & Ivindo National Park & Gabon & -0.17 & 12.25 & 324 & mature rain forest & TRFO & TMFO \\
\hline G13 (b) & Ivindo National Park & Gabon & -0.17 & 12.25 & 325 & mature rain forest & TRFO & TMFO \\
\hline G14 (b) & Ivindo National Park & Gabon & -0.17 & 12.25 & 326 & mature rain forest & TRFO & TMFO \\
\hline G15 (b) & Ivindo National Park & Gabon & -0.17 & 12.25 & 327 & mature rain forest & TRFO & TMFO \\
\hline G17 (b) & Langoué bai & Gabon & -0.17 & 12,57 & 360 & mature rain forest & TRFO & TMFO \\
\hline G18 (b) & Langoué bai & Gabon & -0.17 & 12.55 & 360 & mature rain forest & TRFO & TMFO \\
\hline G31 (b) & Langoué bai & Gabon & -0.17 & 12.54 & 495 & mature rain forest & TRFO & TMFO \\
\hline G37 (b) & Langoué forest & Gabon & -0.17 & 12.53 & 500 & mature rain forest & TRFO & TMFO \\
\hline G44 (b) & Ivindo National Park & Gabon & -0.17 & 12.53 & 500 & mature rain forest & TRFO & TMFO \\
\hline G36 (b) & Langoué forest & Gabon & -0.17 & 12.54 & 515 & mature rain forest & TRFO & TMFO \\
\hline G41 (b) & Langoué forest & Gabon & -0.18 & 12.54 & 459 & mature rain forest & TRFO & TMFO \\
\hline G35 (b) & Langoué forest & Gabon & -0.18 & 12.54 & 468 & mature rain forest & TRFO & TMFO \\
\hline G38 (b) & Langoué forest & Gabon & -0.18 & 12.54 & 495 & mature rain forest & TRFO & TMFO \\
\hline G42 (b) & Langoué forest & Gabon & -0.18 & 12.54 & 463 & mature rain forest & TRFO & TMFO \\
\hline G13L (e) & Lopé National Park & Gabon & -0.18 & 11.59 & 250 & savanna & SAV & SAVA \\
\hline G33 (b) & Langoué bai & Gabon & -0.18 & 12.54 & 460 & mature rain forest & TRFO & TMFO \\
\hline G39 (b) & Langoué forest & Gabon & -0.18 & 12.54 & 455 & mature rain forest & TRFO & TMFO \\
\hline G11L (e) & Lopé National Park & Gabon & -0.18 & 11.67 & 250 & mature rain forest & TRFO & TMFO \\
\hline G25 (b) & Ivindo (camp) & Gabon & -0.18 & 12.54 & 460 & mature rain forest & TRFO & TMFO \\
\hline G40 (b) & Langoué forest & Gabon & -0.18 & 12.54 & 460 & mature rain forest & TRFO & TMFO \\
\hline G29 (b) & Langoué bai & Gabon & -0.18 & 12.50 & 350 & mature rain forest & TRFO & TMFO \\
\hline G28 (b) & Langoué bai & Gabon & -0.19 & 12.50 & 342 & mature rain forest & TRFO & TMFO \\
\hline G32 (b) & Langoué bai & Gabon & -0.19 & 12,54 & 450 & mature rain forest & TRFO & TMFO \\
\hline G30 (b) & Langoué bai & Gabon & -0.19 & 12.50 & 360 & mature rain forest & TRFO & TMFO \\
\hline G34 (b) & Langoué bai & Gabon & -0.19 & 12.54 & 380 & mature rain forest & TRFO & TMFO \\
\hline G43 (b) & Langoué forest & Gabon & -0.19 & 12.54 & 461 & mature rain forest & TRFO & TMFO \\
\hline G27 (b) & Langoué bai & Gabon & -0.19 & 12.55 & 370 & mature rain forest & TRFO & TMFO \\
\hline G10L (e) & Lopé National Park & Gabon & -0.19 & 11.59 & 250 & mature rain forest & TRFO & TMFO \\
\hline G17L (e) & Lopé National Park & Gabon & $-0,19$ & 11.57 & 350 & mature rain forest & TRFO & TMFO \\
\hline G18L (e) & Lopé National Park & Gabon & -0.19 & 11.58 & 300 & mature rain forest & TRFO & TMFO \\
\hline GA9L (e) & Lopé National Park & Gabon & -0.20 & 11.58 & 250 & mature rain forest & TRFO & TMFO \\
\hline
\end{tabular}


Table 1. Continued.

\begin{tabular}{|c|c|c|c|c|c|c|c|c|}
\hline Samples & Location & Country & Lat. & Long. & Alt (m) & Local observed vegetation & Reconstructed biomes & Reconstructed stages \\
\hline G12L (e) & Lopé National Park & Gabon & -0.20 & 11.68 & 250 & mature rain forest & TRFO & TMFO \\
\hline G15L (e) & Lopé National Park & Gabon & -0.20 & 11.56 & 450 & mature rain forest & TRFO & TMFO \\
\hline G16L (e) & Lopé National Park & Gabon & $-0,20$ & 11,58 & 400 & mature rain forest & TRFO & TMFO \\
\hline G14L (e) & Lopé National Park & Gabon & $-0,20$ & 11.55 & 500 & mature rain forest & TRFO & TMFO \\
\hline G24 (b) & Lake Nguene & Gabon & -0.21 & 10.50 & 28 & mature rain forest & TRFO & TMFO \\
\hline GA8L (e) & Lopé National Park & Gabon & -0.21 & 11.59 & 250 & mature rain forest & TRFO & TMFO \\
\hline GA6L (e) & Lopé National Park & Gabon & -0.22 & 11.59 & 250 & mature rain forest & TRFO & TMFO \\
\hline GA7L (e) & Lopé National Park & Gabon & -0.23 & 11.59 & 300 & mature rain forest & TRFO & TMFO \\
\hline GA1L (e) & Lopé National Park & Gabon & -0.68 & 11.75 & 350 & mature rain forest & TRFO & TMFO \\
\hline GA2L (e) & Lopé National Park & Gabon & -0.68 & 11.79 & 330 & mature rain forest & TRFO & TMFO \\
\hline GA3L (e) & Lopé National Park & Gabon & -0.68 & 11.73 & 320 & mature rain forest & TRFO & TMFO \\
\hline GA4L (e) & Lopé National Park & Gabon & -0.68 & 11.78 & 350 & mature rain forest & TRFO & TMFO \\
\hline GA5L (e) & Lopé National Park & Gabon & -0.69 & 11.79 & 350 & mature rain forest & TRFO & TMFO \\
\hline G1 (b) & Lambaréné-Fougamou & Gabon & -0.94 & 10.47 & 148 & mature rain forest & TRFO & TMFO \\
\hline G2 (b) & Lambaréné-Fougamou & Gabon & -0.94 & 10.47 & 148 & mature rain forest & TRFO & TMFO \\
\hline G3 (b) & Lambaréné-Fougamou & Gabon & -0.94 & 10.47 & 148 & mature rain forest & TRFO & TMFO \\
\hline G4 (b) & Doubou & Gabon & -1.75 & 10.87 & 89 & savanna & SAV & SAVA \\
\hline G5 (b) & Doubou & Gabon & -1.75 & 10.87 & 89 & savanna & SAV & SAVA \\
\hline G6 (b) & Doubou & Gabon & -1.75 & 10,87 & 89 & savanna & SAV & SAVA \\
\hline G11 (b) & Bengui & Gabon & -2.02 & 11.11 & 92 & young secondary rain forest & TRFO & TYSF \\
\hline G8 (b) & Biendi & Gabon & -2.06 & 11.07 & 139 & savanna & SAV & SAVA \\
\hline G10 (b) & Lake Massou & Gabon & -2.19 & 11.20 & 134 & savanna & SAV & SAVA \\
\hline G9 (b) & Sogadel & Gabon & -2.44 & 11.42 & 153 & savanna & SAV & SAVA \\
\hline $\mathrm{CO} 29(\mathrm{f})$ & Mandzi & Congo & -4.08 & 12.15 & 200 & mature rain forest & TRFO & TMFO \\
\hline $\operatorname{CO} 30(f)$ & Mandzi & Congo & -4.08 & 12.15 & 200 & mature rain forest & TRFO & TMFO \\
\hline $\operatorname{CO} 31(\mathrm{f})$ & Mandzi & Congo & -4.08 & 12.15 & 200 & mature rain forest & TRFO & TMFO \\
\hline $\operatorname{CO} 32(\mathrm{f})$ & Mandzi & Congo & -4.08 & 12.15 & 200 & mature rain forest & TRFO & TMFO \\
\hline $\operatorname{CO} 33(f)$ & Mandzi & Congo & -4.08 & 12.15 & 200 & mature rain forest & TRFO & TMFO \\
\hline $\mathrm{CO} 34(\mathrm{f})$ & Mandzi & Congo & -4.08 & 12.15 & 200 & mature rain forest & TRFO & TMFO \\
\hline $\operatorname{CO} 35$ (f) & Mandzi & Congo & -4.08 & 12.15 & 200 & mature rain forest & TRFO & TMFO \\
\hline $\mathrm{CO} 36(\mathrm{f})$ & Mandzi & Congo & -4.08 & 12.15 & 200 & mature rain forest & TRFO & TMFO \\
\hline $\operatorname{CO} 37(f)$ & Mandzi & Congo & -4.08 & 12.15 & 200 & mature rain forest & TRFO & TMFO \\
\hline $\mathrm{CO} 1$ (f) & La Tour & Congo & -4.17 & 12.38 & 360 & mature rain forest & TRFO & TMFO \\
\hline $\mathrm{CO} 2$ (f) & La Tour & Congo & -4.17 & 12.38 & 360 & mature rain forest & TRFO & TMFO \\
\hline $\mathrm{CO} 3$ (f) & La Tour & Congo & $-4,17$ & 12.38 & 360 & mature rain forest & TRFO & TMFO \\
\hline $\mathrm{CO} 4(\mathrm{f})$ & La Tour & Congo & -4.17 & 12.38 & 360 & mature rain forest & TRFO & TMFO \\
\hline $\operatorname{CO} 5$ (f) & La Tour & Congo & -4.17 & 12.38 & 360 & mature rain forest & TRFO & TMFO \\
\hline $\operatorname{CO6}(f)$ & La Tour & Congo & -4.17 & 12.38 & 360 & mature rain forest & TRFO & TMFO \\
\hline $\mathrm{CO} 7$ (f) & La Tour & Congo & -4.17 & 12.38 & 360 & mature rain forest & TRFO & TMFO \\
\hline $\operatorname{CO} 8$ (f) & La Tour & Congo & -4.17 & 12.38 & 360 & mature rain forest & TRFO & TMFO \\
\hline $\mathrm{CO} 9$ (f) & La Tour & Congo & -4.17 & 12.38 & 360 & mature rain forest & TRFO & TMFO \\
\hline $\mathrm{CO} 10(\mathrm{f})$ & La Tour & Congo & -4.17 & 12.38 & 360 & mature rain forest & TRFO & TMFO \\
\hline $\mathrm{CO} 41$ (f) & Mpassi Mpassi & Congo & -4.17 & 12.5 & 350 & mature rain forest & TRFO & TMFO \\
\hline
\end{tabular}


Table 1. Continued.

\begin{tabular}{|c|c|c|c|c|c|c|c|c|}
\hline Samples & Location & Country & Lat. & Long. & Alt (m) & Local observed vegetation & Reconstructed biomes & Reconstructed stages \\
\hline $\mathrm{CO} 11$ (f) & Dimonika & Congo & -4.22 & 12.43 & 380 & mature rain forest & TRFO & TMFO \\
\hline $\mathrm{CO} 12$ (f) & Dimonika & Congo & -4.22 & 12.43 & 380 & mature rain forest & TRFO & TMFO \\
\hline $\mathrm{CO} 13(\mathrm{f})$ & Dimonika & Congo & -4.22 & 12.43 & 380 & mature rain forest & TRFO & TMFO \\
\hline $\mathrm{CO} 14(\mathrm{f})$ & Dimonika & Congo & -4.22 & 12.43 & 380 & mature rain forest & TRFO & TMFO \\
\hline $\mathrm{CO} 15$ (f) & Dimonika & Congo & -4.22 & 12.43 & 380 & mature rain forest & TRFO & TMFO \\
\hline $\mathrm{CO} 16$ (f) & Mindou & Congo & -4.28 & 12.07 & 90 & mature rain forest & TRFO & TMFO \\
\hline $\mathrm{CO} 17$ (f) & Mindou & Congo & -4.28 & 12.07 & 90 & mature rain forest & TRFO & TMFO \\
\hline $\mathrm{CO} 18$ (f) & Mindou & Congo & -4.28 & 12.07 & 90 & mature rain forest & TRFO & TMFO \\
\hline $\operatorname{CO} 19$ (f) & Mindou & Congo & -4.28 & 12.07 & 90 & mature rain forest & TRFO & TMFO \\
\hline $\mathrm{CO} 20(\mathrm{f})$ & Mindou & Congo & -4.28 & 12.07 & 90 & mature rain forest & TRFO & TMFO \\
\hline $\mathrm{CO} 21$ (f) & Mindou & Congo & -4.28 & 12.07 & 90 & mature rain forest & TRFO & TMFO \\
\hline $\mathrm{CO} 22$ (f) & Mindou & Congo & -4.28 & 12.07 & 90 & mature rain forest & TRFO & TMFO \\
\hline $\mathrm{CO} 23$ (f) & Mindou & Congo & -4.28 & 12.07 & 90 & mature rain forest & TRFO & TMFO \\
\hline $\mathrm{CO} 24$ (f) & Mindou & Congo & -4.28 & 12.07 & 90 & mature rain forest & TRFO & TMFO \\
\hline $\mathrm{CO} 25(\mathrm{f})$ & Mindou & Congo & -4.28 & 12.07 & 90 & mature rain forest & TRFO & TMFO \\
\hline $\mathrm{CO} 26$ (f) & Mindou & Congo & -4.28 & 12.07 & 90 & mature rain forest & TRFO & TMFO \\
\hline $\mathrm{CO} 27$ (f) & Mindou & Congo & -4.28 & 12.07 & 90 & mature rain forest & TRFO & TMFO \\
\hline $\mathrm{CO} 28$ (f) & Mindou & Congo & -4.28 & 12.07 & 90 & mature rain forest & TRFO & TMFO \\
\hline $\mathrm{CO} 42(\mathrm{f})$ & Les Bandas & Congo & -4.28 & 12.58 & 350 & mature rain forest & TRFO & TMFO \\
\hline $\mathrm{CO} 43$ (f) & Les Bandas & Congo & -4.28 & 12.58 & 350 & mature rain forest & TRFO & TMFO \\
\hline $\operatorname{CO} 39$ (f) & Kitina & Congo & -4.32 & 12.2 & 115 & mature rain forest & TRFO & TMFO \\
\hline $\mathrm{CO} 40$ (f) & Kitina & Congo & -4.32 & 12.2 & 115 & mature rain forest & TRFO & TMFO \\
\hline $\operatorname{CO} 38$ (f) & Les Saras & Congo & -4.35 & 12.32 & 390 & mature rain forest & TRFO & TMFO \\
\hline
\end{tabular}

important in the canopy. The Guineo-Congolian wet rain forest represents the climax ecosystem of the central part of the study area. The upper stratum of this formation is generally $35-45 \mathrm{~m}$ high and is well-distributed in diameter classes. The high canopy density precludes the development of an herbaceous strata and favours epiphytes. The thermal gradient in the canopy is very marked, while the atmospheric moisture is permanently high. This type of forest does not show any noticeable seasonal behaviour (Mayaux et al., 1997, 1999).

2.2 The drier types of Guineo-Congolian rain forest ("semi-deciduous rain forest", Letouzey, 1968, 1985)

These forests, also called dry peripheral semi-evergreen rain forest by White (1983), are located at the border of the wet rain forest and are floristically richer than the previous ones. More individuals of the common largest tree species are deciduous (up to $70 \%$ in the upper stratum) and lose their leaves during the dry season allowing the development of a continuous shrub stratum. The diameter classes' distribution of the upper layer is irregular. The thermal gradient is less marked than in the previous type, while the seasonality is more marked in mesological conditions (Mayaux et al., 1997, 1999).

Secondary forest, occurring on past cultivated areas, is widespread in the region, and corresponds to various stages of forest regrowth in which light-demanding species and pioneers are abundant (e.g. Kahn, 1982; Catinot et al., 1983; White, 1983; Mayaux et al., 1997, 1999). The upper layer of the secondary formations is continuous and homogenous and often characterised by a monospecific composition in its earliest stages, with heliophytic and fast height growth pioneer species.

\subsection{The mosaic of rain forest and secondary grassland}

Much of the rain forest at the northern and southern limits of the Guineo-Congolian region has been destroyed by cultivation and fire and replaced by secondary grassland which often occurs in mosaic with small, usually severely degraded, patches of the original forest.

Inside the Guineo-Congolian domain appear vast savannas, either as large patches surrounding the forest massif, or as small islands enclosed within the forest. The trees and shrubs of these savannas are sparse while grasses form a 
Table 2. Plant functional types proposed for the Atlantic Central African areas under investigations for biome reconstructions and corresponding tropical ecosystems.

\begin{tabular}{ll}
\hline Codes & Plant functional types \\
\hline Te1 & wet tropical evergreen trees and shrubs \\
Te2 & dry tropical evergreen trees and shrubs \\
Tr1 & wet tropical raingreen trees and shrubs \\
Tr2 & dry tropical raingreen trees and shrubs \\
Tr3 & driest tropical raingreen trees and shrubs \\
TLw & wet tropical lianas \\
TLd & dry tropical lianas \\
THw & wet tropical herbs \\
THd & dry tropical herbs \\
g & grasses \\
\hline
\end{tabular}

continuous and high stratum. They find their origin in soil conditions (poorly developed, sandy or lateritic soils), in past and present human activity (settlements, fire) or in past climatic changes (e.g. Robyns, 1936; Richards, 1952; Schwartz et al., 2000).

\section{The modern pollen data set}

A total of 199 modern pollen spectra were compiled in a data set. They all have been exclusively extracted from surface soil (in savanna) or litter (in forest) samples collected following the Wright method (1967) widely used in African modern pollen studies (eg. Jolly et al., 1996; Lézine and Edorh, 1991; Bonnefille et al., 1993 ; Vincens et al., 1997, 2000; Elenga et al., 2000b). The location of the study modern pollen samples is given in Fig. 1 and Table 1. Seventy three samples were collected in southern Cameroon (Vincens et al., 2000 and unpublished data; Lebamba et al., 2009), eighty three in Gabon (Jolly et al., 1996; Lebamba et al., 2009; Ngomanda, unpublished data) and forty three in southern Congo (Elenga et al., 2000b). All samples come from vegetation formations occurring on well-drained soils excluding riparian and swampy formations since these formations are not directly linked to climate but rather to local hydrological conditions. They cover the three main White's vegetation types described above. Local vegetation at each site, extracted from field observations or detailed inventories, is given in Table 1.

The pollen data set comprises a total of 272 pollen taxa which nomenclature was standardized following the list of taxa available in Vincens et al. (2007) and on the African Pollen Data base web site (2008). This list refers to the botanical nomenclature proposed by Lebrun and Stork (1991-1997).

\section{The biomisation method and its application to the modern pollen data set}

The biomisation method classifies the plant taxa represented in the pollen assemblages into a number of plant functional types (PFTs) which are broad classes of plants defined by life form (e.g. tree/shrub/lianas/herbs), leaf form (e.g. broadleaved/needle-leaved), phenology (e.g. evergreen/deciduous) and bioclimatic factors. Pollen taxa are assigned to one or more PFTs, then affinity scores are calculated for each biome in turn based on its list of characteristic PFTs. The pollen sample is assigned to the biome to which it has the highest affinity. This method, initially developed for Europe and now used worldwide, was described in detail by Prentice et al. (1996).

In this paper the biomisation procedure has been applied on our modern pollen data set from Atlantic Central Africa, following two ways: (1) the reconstruction of modern potential biomes (see 4.1) and (2) the reconstruction of potential succession stages of forest regeneration (see 4.2) Comparisons with local or more regional botanical data were performed in the aim to test the level of confidence of our reconstructions, and particularly of our taxa-PFT assignments.

\subsection{Potential biome reconstructions}

The taxon versus site matrix used for these reconstructions include 245 pollen taxa among the 272 identified in the 199 spectra, corresponding to native and non-edaphic pollen taxa. We removed marshy or aquatic herbs and shrubs (Cyperaceae, Burnatia, Sesbania, Typha, Eriocaulaceae, Mimosa pigra, Utricularia, Hydrocotyle), typical swamp trees such (Symphonia globulifera, Phoenix reclinata, Raphia, Pandanus, Rhizophora, Morelia senegalensis, Clappertonia), anthropogenic taxa (Cassia didymobotrya, Ricinus communis, Capsicum, Zea mais, Eucalyptus, Plantago and Elaeis guineensis), all the Pteridophyta which generally were not identified at a high level of determination (genus or species), but also pioneer taxa which distribution is not primarily related to climate (Musanga, Anthocleista, Vismia guineensis and Polyscias fulva).

Compared to previous works undertaken in Africa, we propose in this paper the creation of new PFTs taking into account: (1) a more precise definition of the life form of plants which produce the pollen taxa (trees or shrubs, lianas and herbs) and (2) the place these plants occupy in the different central African ecosystems (e.g. tropical wet rain forest, tropical dry rain forest forest...), mainly linked to bioclimatic factors (e.g. rainfall, temperature, cloud cover, atmospheric humidity...). We have differentiated the trees and shrubs from the lianas and herbs. In the PFTs Te1 (wet tropical 
Table 3. Allocation of the pollen taxa derived from all sites listed in Table 1 to the plant functional types used for the biomes reconstructions.

\begin{tabular}{|c|c|c|c|c|c|c|c|c|c|c|c|}
\hline Family & Taxa & $\mathrm{Te} 1$ & $\mathrm{Te} 2$ & $\operatorname{Tr} 1$ & $\operatorname{Tr} 2$ & $\operatorname{Tr} 3$ & TLw & TLd & THw & THd & $\mathrm{g}$ \\
\hline ACANTHACEAE & Acanthaceae undiff. & $\mathrm{x}$ & $\mathrm{x}$ & $\mathrm{x}$ & $\mathrm{x}$ & $\mathrm{x}$ & $\mathrm{x}$ & & $\mathrm{x}$ & $\mathrm{x}$ & \\
\hline ACANTHACEAE & Anisotes & & & $\mathrm{x}$ & & & & & & & \\
\hline ACANTHACEAE & Asystasia gangetica-type & & & & & & & & $\mathrm{x}$ & & \\
\hline ACANTHACEAE & Mendoncia & & & & & & $\mathrm{x}$ & & & & \\
\hline ACANTHACEAE & Thomandersia & $\mathrm{x}$ & $\mathrm{x}$ & $\mathrm{x}$ & & & & & & & \\
\hline AMARANTHACEAE & Achyranthes-type aspera & & & & & & & & & $\mathrm{x}$ & \\
\hline AMARANTHACEAE & Cyathula-type & & & & & & & & $\mathrm{x}$ & $\mathrm{x}$ & \\
\hline AMARANTHACEAE & Sericostachys-type scandens & & & & & & $\mathrm{x}$ & & & & \\
\hline AMARANTHACEAE/CHENOPODIACEAE & Amaranthaceae/Chenopodiaceae undiff. & & & & & & $\mathrm{x}$ & & $\mathrm{x}$ & $\mathrm{x}$ & \\
\hline ANACARDIACEAE & Anacardiaceae undiff. & $\mathrm{x}$ & $\mathrm{x}$ & $\mathrm{x}$ & $\mathrm{x}$ & $\mathrm{x}$ & $\mathrm{x}$ & & & & \\
\hline ANACARDIACEAE & Antrocaryon-type & $\mathrm{x}$ & $\mathrm{x}$ & $\mathrm{x}$ & & & & & & & \\
\hline ANACARDIACEAE & Antrocaryon-type klaineanum & & & $\mathrm{x}$ & & & & & & & \\
\hline ANACARDIACEAE & Fegimanra & & & & $\mathrm{x}$ & & & & & & \\
\hline ANACARDIACEAE & Lannea-type & & $\mathrm{x}$ & $\mathrm{x}$ & $\mathrm{x}$ & $\mathrm{x}$ & & & & & \\
\hline ANACARDIACEAE & Pseudospondias-type & $\mathrm{x}$ & $\mathrm{x}$ & & & & & & & & \\
\hline ANACARDIACEAE & Sorindeia-type & $\mathrm{x}$ & $\mathrm{x}$ & $\mathrm{x}$ & & & & & & & \\
\hline ANACARDIACEAE & Trichoscypha-type & $\mathrm{x}$ & $\mathrm{x}$ & $\mathrm{x}$ & & & $\mathrm{x}$ & & & & \\
\hline ANISOPHYLLEACEAE & Anisophyllea & $\mathrm{x}$ & & & & & & & & & \\
\hline ANISOPHYLLEACEAE & Anopyxis klaineana & $\mathrm{x}$ & $\mathrm{x}$ & & & & & & & & \\
\hline ANNONACEAE & Annonaceae undiff. & $\mathrm{x}$ & $\mathrm{x}$ & $\mathrm{x}$ & $\mathrm{x}$ & $\mathrm{x}$ & $\mathrm{x}$ & & & & \\
\hline APIACEAE & Apiaceae undiff. & & & & & & & & & $\mathrm{x}$ & \\
\hline APOCYNACEAE & Alstonia-type & $\mathrm{x}$ & $\mathrm{x}$ & & & & & & & & \\
\hline APOCYNACEAE & Alstonia-type boonei & $\mathrm{x}$ & $\mathrm{x}$ & & & & & & & & \\
\hline APOCYNACEAE & Apocynaceae undiff. & $\mathrm{x}$ & $\mathrm{x}$ & $\mathrm{x}$ & $\mathrm{x}$ & $\mathrm{x}$ & $\mathrm{x}$ & $\mathrm{x}$ & & & \\
\hline APOCYNACEAE & Funtumia-type & & $\mathrm{x}$ & $\mathrm{x}$ & & & & & & & \\
\hline APOCYNACEAE & Landolphia-type & & & & & & $\mathrm{x}$ & $\mathrm{x}$ & & & \\
\hline APOCYNACEAE & Oncinotis-type & & & & & & $\mathrm{x}$ & & & & \\
\hline APOCYNACEAE & Picralima-type nitida & $\mathrm{x}$ & $\mathrm{x}$ & $\mathrm{x}$ & & & & & & & \\
\hline APOCYNACEAE & Pleiocarpa & & & $\mathrm{x}$ & & & & & & & \\
\hline APOCYNACEAE & Rauvolfia & $\mathrm{x}$ & $\mathrm{x}$ & $\mathrm{x}$ & & & & & & & \\
\hline APOCYNACEAE & Tabernaemontana & & & $\mathrm{x}$ & & & & & & & \\
\hline ASTERACEAE & Asteraceae undiff. & & & & $\mathrm{x}$ & $\mathrm{x}$ & & & & $\mathrm{x}$ & \\
\hline ASTERACEAE & Vernonieae undiff. & & & & $\mathrm{x}$ & $\mathrm{x}$ & & & & $\mathrm{x}$ & \\
\hline BALANITACEAE & Balanites & $\mathrm{x}$ & $\mathrm{x}$ & $\mathrm{x}$ & & & & & & & \\
\hline BEGONIACEAE & Begonia & & & & & & & & $\mathrm{x}$ & & \\
\hline BOMBACACEAE & Bombacaceae undiff. & & $\mathrm{x}$ & $\mathrm{x}$ & & & & & & & \\
\hline BOMBACACEAE & Ceiba pentandra & & $\mathrm{x}$ & $\mathrm{x}$ & & & & & & & \\
\hline BORAGINACEAE & Boraginaceae undiff. & & & $\mathrm{x}$ & $\mathrm{x}$ & $\mathrm{x}$ & & & & $\mathrm{x}$ & \\
\hline BORAGINACEAE & Cordia platythyrsa-type & & & $\mathrm{x}$ & & & & & & & \\
\hline BORAGINACEAE & Ehretia & & & $\mathrm{x}$ & $\mathrm{x}$ & $\mathrm{x}$ & & & & & \\
\hline BORAGINACEAE & Heliotropium indicum-type & & & & & & & & & $\mathrm{x}$ & \\
\hline BORAGINACEAE & Heliotropium steudneri-type & & & & & & & & & $\mathrm{x}$ & \\
\hline BURSERACEAE & Aucoumea klaineana & $\mathrm{x}$ & & & & & & & & & \\
\hline
\end{tabular}


Table 3. Continued.

\begin{tabular}{|c|c|c|c|c|c|c|c|c|c|c|c|}
\hline Family & Taxa & $\mathrm{Te} 1$ & $\mathrm{Te} 2$ & $\operatorname{Tr} 1$ & $\operatorname{Tr} 2$ & $\operatorname{Tr} 3$ & TLw & TLd & THw & THd & $\mathrm{g}$ \\
\hline BURSERACEAE & Burseraceae undiff. & $\mathrm{x}$ & $\mathrm{x}$ & $\mathrm{x}$ & & $\mathrm{x}$ & & & & & \\
\hline BURSERACEAE & Canarium-type & $\mathrm{x}$ & $\mathrm{x}$ & $\mathrm{x}$ & & & & & & & \\
\hline BURSERACEAE & Commiphora edulis-type & & & & & $\mathrm{x}$ & & & & & \\
\hline BURSERACEAE & Dacryodes-type & $\mathrm{x}$ & $\mathrm{x}$ & & & & & & & & \\
\hline BURSERACEAE & Santiria-type & $\mathrm{x}$ & $\mathrm{x}$ & & & & & & & & \\
\hline CAESALPINIACEAE & Anthonotha-type & $\mathrm{x}$ & $\mathrm{x}$ & $\mathrm{x}$ & & & & & & & \\
\hline CAESALPINIACEAE & Berlinia-type & $\mathrm{x}$ & $\mathrm{x}$ & $\mathrm{x}$ & & & & & & & \\
\hline CAESALPINIACEAE & Caesalpiniaceae undiff. & $\mathrm{x}$ & $\mathrm{x}$ & & & & $\mathrm{x}$ & & & & \\
\hline CAESALPINIACEAE & Copaifera-type & $\mathrm{x}$ & $\mathrm{x}$ & & & & & & & & \\
\hline CAESALPINIACEAE & Crudia-type gabonensis & $\mathrm{x}$ & & & & & & & & & \\
\hline CAESALPINIACEAE & Daniellia & $\mathrm{x}$ & & & $\mathrm{x}$ & $\mathrm{x}$ & & & & & \\
\hline CAESALPINIACEAE & Detarium & $\mathrm{x}$ & $\mathrm{x}$ & $\mathrm{x}$ & $\mathrm{x}$ & & & & & & \\
\hline CAESALPINIACEAE & Dialium & $\mathrm{x}$ & $\mathrm{x}$ & $\mathrm{x}$ & & $\mathrm{x}$ & & & & & \\
\hline CAESALPINIACEAE & Dialium pachyphyllum-type & $\mathrm{x}$ & & & & & & & & & \\
\hline CAESALPINIACEAE & Distemonanthus benthamianus-type & $\mathrm{x}$ & $\mathrm{x}$ & $\mathrm{x}$ & & & & & & & \\
\hline CAESALPINIACEAE & Duparquetia orchidacea & & & & & $\mathrm{x}$ & & & & & \\
\hline CAESALPINIACEAE & Gilbertiodendron-type & $\mathrm{x}$ & & & & & & & & & \\
\hline CAESALPINIACEAE & Guibourtia & $\mathrm{x}$ & & & & & & & & & \\
\hline CAESALPINIACEAE & Guibourtia demeusei-type & $\mathrm{x}$ & & & & & & & & & \\
\hline CAESALPINIACEAE & Hylodendron gabunense & $\mathrm{x}$ & $\mathrm{x}$ & $\mathrm{x}$ & & & & & & & \\
\hline CAESALPINIACEAE & Hymenostegia-type pellegrinii & $\mathrm{x}$ & & & & & & & & & \\
\hline CAESALPINIACEAE & Tessmannia & $\mathrm{x}$ & & & & & & & & & \\
\hline CAPPARIDACEAE & Capparidaceae undiff. & $\mathrm{x}$ & $\mathrm{x}$ & $\mathrm{x}$ & $\mathrm{x}$ & $\mathrm{x}$ & & & & $\mathrm{x}$ & \\
\hline CELASTRACEAE/HIPPOCRATEACEAE & Celastraceae/Hippocrateaceae undiff. & $\mathrm{x}$ & $\mathrm{x}$ & $\mathrm{x}$ & $\mathrm{x}$ & $\mathrm{x}$ & $\mathrm{x}$ & $\mathrm{x}$ & & & \\
\hline CHRYSOBALANACEAE & Chrysobalanus-type icaco & $\mathrm{x}$ & & & & & & & & & \\
\hline CHRYSOBALANACEAE & Maranthes-type & $\mathrm{x}$ & $\mathrm{x}$ & $\mathrm{x}$ & & & & & & & \\
\hline CHRYSOBALANACEAE & Parinari-type & $\mathrm{x}$ & $\mathrm{x}$ & $\mathrm{x}$ & $\mathrm{x}$ & $\mathrm{x}$ & & & & & \\
\hline CLUSIACEAE & Allanblackia & $\mathrm{x}$ & & & & & & & & & \\
\hline CLUSIACEAE & Garcinia epunctata-type & $\mathrm{x}$ & $\mathrm{x}$ & $\mathrm{x}$ & & & & & & & \\
\hline CLUSIACEAE & Mammea africana-type & $\mathrm{x}$ & $\mathrm{x}$ & $\mathrm{x}$ & & & & & & & \\
\hline COMBRETACEAE & Combretaceae undiff. & $\mathrm{x}$ & $\mathrm{x}$ & $\mathrm{x}$ & $\mathrm{x}$ & $\mathrm{x}$ & $\mathrm{x}$ & & & & \\
\hline COMBRETACEAE & Terminalia-type & $\mathrm{x}$ & $\mathrm{x}$ & $\mathrm{x}$ & $\mathrm{x}$ & $\mathrm{x}$ & & & & & \\
\hline COMBRETACEAE/MELASTOMATACEAE & Combretaceae/Melastomataceae undiff. & $\mathrm{x}$ & $\mathrm{x}$ & $\mathrm{x}$ & $\mathrm{x}$ & $\mathrm{x}$ & $\mathrm{x}$ & & $\mathrm{x}$ & $\mathrm{x}$ & \\
\hline CONNARACEAE & Cnestis & $\mathrm{x}$ & $\mathrm{x}$ & $\mathrm{x}$ & & & $\mathrm{x}$ & & & & \\
\hline CONVOLVULACEAE & Convolvulaceae undiff. & & & & & & $\mathrm{x}$ & & $\mathrm{x}$ & $\mathrm{x}$ & \\
\hline CONVOLVULACEAE & Evolvulus-type & & & & & & & & & $\mathrm{x}$ & \\
\hline CONVOLVULACEAE & Ipomoea-type & & & & & & & & $\mathrm{x}$ & $\mathrm{x}$ & \\
\hline CUCURBITACEAE & Coccinia & & & & & & & & & $\mathrm{x}$ & \\
\hline CUCURBITACEAE & Cucurbitaceae undiff. & & & & & & & & $\mathrm{x}$ & $\mathrm{x}$ & \\
\hline CUCURBITACEAE & Luffa-type & & & & & & & & & $\mathrm{x}$ & \\
\hline DILLENIACEAE & Tetracera & $\mathrm{x}$ & $\mathrm{x}$ & $\mathrm{x}$ & & & $\mathrm{x}$ & & & & \\
\hline DIOSCOREACEAE & Dioscorea & & & & & & & & $\mathrm{x}$ & $\mathrm{x}$ & \\
\hline
\end{tabular}


Table 3. Continued.

\begin{tabular}{|c|c|c|c|c|c|c|c|c|c|c|}
\hline Family & Taxa & $\mathrm{Te} 1$ & $\mathrm{Te} 2$ & $\operatorname{Tr} 1$ & $\operatorname{Tr} 2$ & $\operatorname{Tr} 3$ & TLw & TLd & $\mathrm{THw}$ & THd \\
\hline DRACAENACEAE & Dracaena & $\mathrm{x}$ & $\mathrm{x}$ & $\mathrm{x}$ & $\mathrm{x}$ & $\mathrm{x}$ & & & & \\
\hline EBENACEAE & Diospyros & $\mathrm{x}$ & $\mathrm{x}$ & & & & & & & \\
\hline EUPHORBIACEAE & Acalypha & & & $\mathrm{x}$ & $\mathrm{x}$ & $\mathrm{x}$ & & & & $\mathrm{x}$ \\
\hline EUPHORBIACEAE & Alchornea & $\mathrm{x}$ & $\mathrm{x}$ & $\mathrm{x}$ & & & & & & \\
\hline EUPHORBIACEAE & Anthostema-type & $\mathrm{x}$ & & & & & & & & \\
\hline EUPHORBIACEAE & Antidesma-type & $\mathrm{x}$ & $\mathrm{x}$ & $\mathrm{x}$ & $\mathrm{x}$ & $\mathrm{x}$ & & & & \\
\hline EUPHORBIACEAE & Bridelia ferruginea-type & & & $\mathrm{x}$ & $\mathrm{x}$ & $\mathrm{x}$ & & & & \\
\hline EUPHORBIACEAE & Bridelia micrantha-type & $\mathrm{x}$ & $\mathrm{x}$ & $\mathrm{x}$ & $\mathrm{x}$ & $\mathrm{x}$ & & & & \\
\hline EUPHORBIACEAE & Centroplacus glaucinus & $\mathrm{x}$ & $\mathrm{x}$ & $\mathrm{x}$ & & & & & & \\
\hline EUPHORBIACEAE & Cleistanthus-type polystachyus & $\mathrm{x}$ & $\mathrm{x}$ & & & & & & & \\
\hline EUPHORBIACEAE & Croton-type & & & $\mathrm{x}$ & & & & & & \\
\hline EUPHORBIACEAE & Cyathogyne & $\mathrm{x}$ & & & & & & & $\mathrm{x}$ & \\
\hline EUPHORBIACEAE & Cyttaranthus congolensis & $\mathrm{x}$ & $\mathrm{x}$ & $\mathrm{x}$ & & & & & & \\
\hline EUPHORBIACEAE & Discoglypremna caloneura & $\mathrm{x}$ & $\mathrm{x}$ & $\mathrm{x}$ & & & & & & \\
\hline EUPHORBIACEAE & Drypetes-type & $\mathrm{x}$ & $\mathrm{x}$ & $\mathrm{x}$ & & & & & & \\
\hline EUPHORBIACEAE & Elaeophorbia-type & & & $\mathrm{x}$ & & & & & & \\
\hline EUPHORBIACEAE & Euphorbia-type & & & & & & & & & $\mathrm{x}$ \\
\hline EUPHORBIACEAE & Euphorbiaceae undiff. & $\mathrm{x}$ & $\mathrm{x}$ & $\mathrm{x}$ & $\mathrm{x}$ & $\mathrm{x}$ & $\mathrm{x}$ & & $\mathrm{x}$ & $\mathrm{x}$ \\
\hline EUPHORBIACEAE & Klaineanthus gaboniae & $\mathrm{x}$ & & & & & & & & \\
\hline EUPHORBIACEAE & Macaranga-type & $\mathrm{x}$ & $\mathrm{x}$ & $\mathrm{x}$ & & & & & & \\
\hline EUPHORBIACEAE & Mallotus-type oppositifolius & $\mathrm{x}$ & $\mathrm{x}$ & $\mathrm{x}$ & & & & & & \\
\hline EUPHORBIACEAE & Margaritaria discoidea & $\mathrm{x}$ & $\mathrm{x}$ & $\mathrm{x}$ & & & & & & \\
\hline EUPHORBIACEAE & Martretia quadricornis & $\mathrm{x}$ & & & & & & & & \\
\hline EUPHORBIACEAE & Phyllanthus-type & $\mathrm{x}$ & $\mathrm{x}$ & $\mathrm{x}$ & $\mathrm{x}$ & $\mathrm{x}$ & & & $\mathrm{x}$ & $\mathrm{x}$ \\
\hline EUPHORBIACEAE & Plagiostyles-type africana & $\mathrm{x}$ & & & & & & & & \\
\hline EUPHORBIACEAE & Tetrorchidium & $\mathrm{x}$ & $\mathrm{x}$ & $\mathrm{x}$ & & & & & & \\
\hline EUPHORBIACEAE & Uapaca & $\mathrm{x}$ & $\mathrm{x}$ & $\mathrm{x}$ & & & & & & \\
\hline EUPHORBIACEAE & Uapaca guineensis-type & $\mathrm{x}$ & $\mathrm{x}$ & $\mathrm{x}$ & & & & & & \\
\hline EUPHORBIACEAE & Uapaca heudelotii-type & $\mathrm{x}$ & $\mathrm{x}$ & $\mathrm{x}$ & & & & & & \\
\hline FABACEAE & Baphia-type & $\mathrm{x}$ & $\mathrm{x}$ & $\mathrm{x}$ & & & & & & \\
\hline FABACEAE & Aeschynomene baumii-type & & & & $\mathrm{x}$ & $\mathrm{x}$ & & & & \\
\hline FABACEAE & Fabaceae undiff. & $\mathrm{x}$ & $\mathrm{x}$ & $\mathrm{x}$ & $\mathrm{x}$ & $\mathrm{x}$ & $\mathrm{x}$ & $\mathrm{x}$ & $\mathrm{x}$ & $\mathrm{x}$ \\
\hline FABACEAE & Indigofera & & & & & & & & & $\mathrm{x}$ \\
\hline FABACEAE & Pterocarpus-type & $\mathrm{x}$ & $\mathrm{x}$ & $\mathrm{x}$ & $\mathrm{x}$ & $\mathrm{x}$ & & & & \\
\hline FLACOURTIACEAE & Caloncoba-type & $\mathrm{x}$ & $\mathrm{x}$ & $\mathrm{x}$ & $\mathrm{x}$ & $\mathrm{x}$ & & & & \\
\hline FLACOURTIACEAE & Camptostylus & $\mathrm{x}$ & $\mathrm{x}$ & $\mathrm{x}$ & & & & & & \\
\hline FLACOURTIACEAE & Casearia & $\mathrm{x}$ & $\mathrm{x}$ & $\mathrm{x}$ & $\mathrm{x}$ & & & & & \\
\hline FLACOURTIACEAE & Flacourtiaceae undiff. & $\mathrm{x}$ & $\mathrm{x}$ & $\mathrm{x}$ & $\mathrm{x}$ & $\mathrm{x}$ & & & & \\
\hline FLACOURTIACEAE & Homalium & $\mathrm{x}$ & $\mathrm{x}$ & $\mathrm{x}$ & & & & & & \\
\hline FLACOURTIACEAE & Scottelia klaineana-type & $\mathrm{x}$ & $\mathrm{x}$ & & & & & & & \\
\hline FLAGELLARIACEAE & Flagellaria & & & & & & & $\mathrm{x}$ & & \\
\hline HYMENOCARDIACEAE & Hymenocardia & & & $\mathrm{x}$ & $\mathrm{x}$ & $\mathrm{x}$ & & & & \\
\hline HYMENOCARDIACEAE & Hymenocardia ulmoides-type & & & $\mathrm{x}$ & $\mathrm{x}$ & & & & & \\
\hline HYPERICACEAE & Harungana madagascariensis-type & & & & $\mathrm{x}$ & $\mathrm{x}$ & & & & \\
\hline
\end{tabular}


Table 3. Continued.

\begin{tabular}{|c|c|c|c|c|c|c|c|c|c|c|c|}
\hline Family & Taxa & $\mathrm{Te} 1$ & $\mathrm{Te} 2$ & $\operatorname{Tr} 1$ & $\operatorname{Tr} 2$ & $\operatorname{Tr} 3$ & TLw & TLd & $\mathrm{THw}$ & THd & $\mathrm{g}$ \\
\hline ICACINACEAE & Icacinaceae undiff. & $\mathrm{x}$ & $\mathrm{x}$ & $\mathrm{x}$ & & & $\mathrm{x}$ & & & & \\
\hline ICACINACEAE & Raphiostylis & $\mathrm{x}$ & & & & & $\mathrm{x}$ & & & & \\
\hline IRVINGIACEAE & Irvingia-type gabonensis & $\mathrm{x}$ & $\mathrm{x}$ & $\mathrm{x}$ & & & & & & & \\
\hline LAMIACEAE & Hoslundia-type opposita & & & & $\mathrm{x}$ & $\mathrm{x}$ & & & & & \\
\hline LAMIACEAE & Lamiaceae undiff. & & & & $\mathrm{x}$ & $\mathrm{x}$ & & & & $\mathrm{x}$ & \\
\hline LECYTHIDACEAE & Petersianthus-type macrocarpus & $\mathrm{x}$ & $\mathrm{x}$ & & & & & & & & \\
\hline LEGUMINOSAE & Leguminosae undiff. & $\mathrm{x}$ & $\mathrm{x}$ & $\mathrm{x}$ & $\mathrm{x}$ & $\mathrm{x}$ & $\mathrm{x}$ & $\mathrm{x}$ & $\mathrm{x}$ & $\mathrm{x}$ & \\
\hline LOGANIACEAE & Strychnos & $\mathrm{x}$ & $\mathrm{x}$ & $\mathrm{x}$ & $\mathrm{x}$ & $\mathrm{x}$ & $\mathrm{x}$ & & & & \\
\hline LORANTHACEAE & Loranthaceae undiff. & $\mathrm{x}$ & $\mathrm{x}$ & $\mathrm{x}$ & $\mathrm{x}$ & $\mathrm{x}$ & & & & & \\
\hline MALPIGHIACEAE & Acridocarpus & & & $\mathrm{x}$ & $\mathrm{x}$ & $\mathrm{x}$ & $\mathrm{x}$ & $\mathrm{x}$ & & & \\
\hline MELASTOMATACEAE & Melastomataceae undiff. & $\mathrm{x}$ & $\mathrm{x}$ & $\mathrm{x}$ & $\mathrm{x}$ & $\mathrm{x}$ & & & $\mathrm{x}$ & $\mathrm{x}$ & \\
\hline MELIACEAE & Carapa-type procera & $\mathrm{x}$ & $\mathrm{x}$ & $\mathrm{x}$ & & & & & & & \\
\hline MELIACEAE & Entandrophragma-type & $\mathrm{x}$ & $\mathrm{x}$ & $\mathrm{x}$ & & & & & & & \\
\hline MELIACEAE & Khaya-type & $\mathrm{x}$ & $\mathrm{x}$ & $\mathrm{x}$ & & & & & & & \\
\hline MELIACEAE & Meliaceae undiff. & $\mathrm{x}$ & $\mathrm{x}$ & $\mathrm{x}$ & & & & & & & \\
\hline MELIACEAE & Trichilia-type & $\mathrm{x}$ & $\mathrm{x}$ & $\mathrm{x}$ & & & & & & & \\
\hline MENISPERMACEAE & Menispermaceae undiff. & & & & & & $\mathrm{x}$ & $\mathrm{x}$ & & & \\
\hline MENISPERMACEAE & Tiliacora-type funifera & & & & & & $\mathrm{x}$ & & & & \\
\hline MIMOSACEAE & Acacia & & & & & $\mathrm{x}$ & $\mathrm{x}$ & & & & \\
\hline MIMOSACEAE & Albizia-type & & & $\mathrm{x}$ & $\mathrm{x}$ & $\mathrm{x}$ & & & & & \\
\hline MIMOSACEAE & Calpocalyx-type & $\mathrm{x}$ & & & & & & & & & \\
\hline MIMOSACEAE & Calpocalyx-type letestui & $\mathrm{x}$ & & & & & & & & & \\
\hline MIMOSACEAE & Cylicodiscus-type gabunensis & $\mathrm{x}$ & & & & & & & & & \\
\hline MIMOSACEAE & Entada-type & & & & $\mathrm{x}$ & $\mathrm{x}$ & $\mathrm{x}$ & $\mathrm{x}$ & G & & \\
\hline MIMOSACEAE & Mimosaceae undiff. & $\mathrm{x}$ & $\mathbf{x}$ & $\mathrm{x}$ & $\mathrm{x}$ & $\mathrm{x}$ & $\mathrm{x}$ & $\mathrm{x}$ & & & \\
\hline MIMOSACEAE & Parkia & & & $\mathrm{x}$ & $\mathrm{x}$ & $\mathrm{x}$ & & & & & \\
\hline MIMOSACEAE & Pentaclethra macrophylla & $\mathrm{x}$ & $\mathrm{x}$ & $\mathrm{x}$ & & & & & & & \\
\hline MIMOSACEAE & Pentaclethra-type eetveldeana & $\mathrm{x}$ & $\mathrm{x}$ & $\mathrm{x}$ & & & & & & & \\
\hline MIMOSACEAE & Piptadeniastrum-type africanum & $\mathrm{x}$ & $\mathrm{x}$ & $\mathrm{x}$ & & & & & & & \\
\hline MIMOSACEAE & Tetrapleura tetraptera-type & $\mathrm{x}$ & $\mathrm{x}$ & $\mathrm{x}$ & & & & & & & \\
\hline MONOCOTYLEDONEAE & Monocotyledoneae undiff. & & & & & & & & $\mathrm{x}$ & $\mathrm{x}$ & \\
\hline MORACEAE & Antiaris-type toxicaria & $\mathrm{x}$ & $\mathrm{x}$ & $\mathrm{x}$ & & & & & & & \\
\hline MORACEAE & Dorstenia-type & & & & & & & & $\mathrm{x}$ & & \\
\hline MORACEAE & Ficus & $\mathrm{x}$ & $\mathrm{x}$ & $\mathrm{x}$ & $\mathrm{x}$ & $\mathrm{x}$ & $\mathrm{x}$ & & & & \\
\hline MORACEAE & Milicia-type excelsa & $\mathrm{x}$ & $\mathrm{x}$ & $\mathrm{x}$ & & & & & & & \\
\hline MORACEAE & Moraceae undiff. & $\mathrm{x}$ & $\mathrm{x}$ & $\mathrm{x}$ & $\mathrm{x}$ & $\mathrm{x}$ & $\mathrm{x}$ & & & & \\
\hline MORACEAE & Myrianthus-type arboreus & & $\mathrm{x}$ & $\mathrm{x}$ & & & & & & & \\
\hline MORACEAE & Treculia & $\mathrm{x}$ & $\mathrm{x}$ & $\mathrm{x}$ & & & & & & & \\
\hline MORACEAE & Trilepisium-type madagascariensis & & & $\mathrm{x}$ & & & & & & & \\
\hline MYRISTICACEAE & Coelocaryon & $\mathrm{x}$ & & & & & & & & & \\
\hline MYRISTICACEAE & Pycnanthus angolensis-type & $\mathrm{x}$ & $\mathrm{x}$ & $\mathrm{x}$ & & & & & & & \\
\hline MYRISTICACEAE & Scyphocephalium & $\mathrm{x}$ & & & & & & & & & \\
\hline MYRISTICACEAE & Staudtia kamerunensis & $\mathrm{x}$ & $\mathrm{x}$ & $\mathrm{x}$ & & & & & & & \\
\hline MYRTACEAE & Syzygium-type & $\mathrm{x}$ & $\mathrm{x}$ & $\mathrm{x}$ & $\mathrm{x}$ & $\mathrm{x}$ & & & & & \\
\hline OCHNACEAE & Campylospermum & $\mathrm{x}$ & $\mathrm{x}$ & $\mathrm{x}$ & & & & & & & \\
\hline OCHNACEAE & Lophira alata-type & $\mathrm{x}$ & & & & & & & & & \\
\hline OLACACEAE & Coula edulis & $\mathrm{x}$ & & & & & & & & & \\
\hline OLACACEAE & Heisteria & $\mathrm{x}$ & $\mathrm{x}$ & $\mathrm{x}$ & & & & & & & \\
\hline OLACACEAE & Olax & $\mathrm{x}$ & $\mathrm{x}$ & $\mathrm{x}$ & & & & & & & \\
\hline OLACACEAE & Strombosia & $\mathrm{x}$ & $\mathrm{x}$ & & & & & & & & \\
\hline OLACACEAE & Strombosia scheffleri-type & $\mathrm{x}$ & $\mathrm{x}$ & & & & & & & & \\
\hline OLACACEAE & Strombosiopsis tetrandra & $\mathrm{x}$ & & & & & & & & & \\
\hline PALMAE & Borassus-type aethiopum & & & & & $\mathrm{x}$ & & & & & \\
\hline PALMAE & Podococcus barteri & $\mathrm{x}$ & & & & & & & & & \\
\hline PALMAE & Sclerosperma & $\mathrm{x}$ & & & & & & & & & \\
\hline PANDACEAE & Microdesmis & $\mathrm{x}$ & $\mathrm{x}$ & $\mathrm{x}$ & & & & & & & \\
\hline POACEAE & Poaceae undiff. & & & & & & & & & & $\mathrm{x}$ \\
\hline RANUNCULACEAE & Clematis-type & & & & & & $\mathrm{x}$ & $\mathrm{x}$ & & & \\
\hline RHAMNACEAE & Rhamnaceae undiff. & $\mathrm{x}$ & $\mathrm{x}$ & $\mathrm{x}$ & $\mathrm{x}$ & $\mathrm{x}$ & $\mathrm{x}$ & & & & \\
\hline
\end{tabular}


Table 3. Continued.

\begin{tabular}{|c|c|c|c|c|c|c|c|c|c|c|c|}
\hline Family & Taxa & $\mathrm{Te} 1$ & $\mathrm{Te} 2$ & $\operatorname{Tr} 1$ & $\operatorname{Tr} 2$ & $\operatorname{Tr} 3$ & TLw & TLd & THw & THd & $\mathrm{g}$ \\
\hline RUBIACEAE & Aidia-type & $\mathrm{x}$ & & & & & & & & & \\
\hline RUBIACEAE & Aidia-type micrantha & $\mathrm{x}$ & & & & & & & & & \\
\hline RUBIACEAE & Crossopteryx febrifuga & & & & $\mathrm{x}$ & $\mathrm{x}$ & & & & & \\
\hline RUBIACEAE & Hallea-type & $\mathrm{x}$ & & & & & & & & & \\
\hline RUBIACEAE & Hallea-type rubrostipulata & $\mathrm{x}$ & & & & & & & & & \\
\hline RUBIACEAE & Hymenodictyon-type floribundum & & & & $\mathrm{x}$ & $\mathrm{x}$ & & & & & \\
\hline RUBIACEAE & Keetia-type gueinzii & & & $\mathrm{x}$ & $\mathrm{x}$ & $\mathrm{x}$ & $\mathrm{x}$ & $\mathrm{x}$ & & & \\
\hline RUBIACEAE & Macrosphyra-type & & & & & $\mathrm{x}$ & & $\mathrm{x}$ & & & \\
\hline RUBIACEAE & Morinda & & & $\mathrm{x}$ & $\mathrm{x}$ & $\mathrm{x}$ & & & & & \\
\hline RUBIACEAE & Nauclea-type & $\mathrm{x}$ & $\mathrm{x}$ & $\mathrm{x}$ & $\mathrm{x}$ & $\mathrm{x}$ & & & & & \\
\hline RUBIACEAE & Oldenlandia-type & & & & & & & & & $\mathrm{x}$ & \\
\hline RUBIACEAE & Oligocodon-type cuniliffeae & & & & & & $\mathrm{x}$ & & & & \\
\hline RUBIACEAE & Pausinystalia-type macroceras & $\mathrm{x}$ & $\mathrm{x}$ & & & & & & & & \\
\hline RUBIACEAE & Psychotria & $\mathrm{x}$ & $\mathrm{x}$ & $\mathrm{x}$ & & & & & & & \\
\hline RUBIACEAE & Psydrax-type schimperiana & $\mathrm{x}$ & & & & & & & & & \\
\hline RUBIACEAE & Psydrax-type subcordata & $\mathrm{x}$ & & & & & & & & & \\
\hline RUBIACEAE & Rubiaceae undiff. & $\mathrm{x}$ & $\mathrm{x}$ & $\mathrm{x}$ & $\mathrm{x}$ & $\mathrm{x}$ & $\mathrm{x}$ & $\mathrm{x}$ & $\mathrm{x}$ & $\mathrm{x}$ & \\
\hline RUBIACEAE & Sherbournia bignoniiflora-type & & & & & & $\mathrm{x}$ & & & & \\
\hline RUBIACEAE & Spermacoce-type & & & & & & & & & $\mathrm{x}$ & \\
\hline RUBIACEAE & Uncaria-type africana & & & & & & $\mathrm{x}$ & & & & \\
\hline RUTACEAE & Rutaceae undiff. & $\mathrm{x}$ & $\mathrm{x}$ & $\mathrm{x}$ & & & $\mathrm{x}$ & & & & \\
\hline RUTACEAE & Vepris-type & $\mathrm{x}$ & $\mathrm{x}$ & $\mathrm{x}$ & & & & & & & \\
\hline RUTACEAE & Zanthoxylum-type & & $\mathrm{x}$ & $\mathrm{x}$ & & & $\mathrm{x}$ & & & & \\
\hline SAPINDACEAE & Allophylus & $\mathrm{x}$ & $\mathrm{x}$ & $\mathrm{x}$ & $\mathrm{x}$ & $\mathrm{x}$ & $\mathrm{x}$ & & & & \\
\hline SAPINDACEAE & Aphania-type senegalensis & $\mathrm{x}$ & $\mathrm{x}$ & $\mathrm{x}$ & & & & & & & \\
\hline SAPINDACEAE & Blighia & $\mathrm{x}$ & $\mathrm{x}$ & $\mathrm{x}$ & $\mathrm{x}$ & $\mathrm{x}$ & & & & & \\
\hline SAPINDACEAE & Cardiospermum & & & & & & $\mathrm{x}$ & $\mathrm{x}$ & & & \\
\hline SAPINDACEAE & Chytranthus-type & $\mathrm{x}$ & & & & & & & & & \\
\hline SAPINDACEAE & Dodonaea & & & & $\mathrm{x}$ & & & & & & \\
\hline SAPINDACEAE & Eriocoelum & $\mathrm{x}$ & & & & & & & & & \\
\hline SAPINDACEAE & Ganophyllum-type giganteum & & $\mathrm{x}$ & $\mathrm{x}$ & & & & & & & \\
\hline SAPINDACEAE & Laccodiscus & $\mathrm{x}$ & & & & & & & & & \\
\hline SAPINDACEAE & Lecaniodiscus-type & & & $\mathrm{x}$ & & & & & & & \\
\hline SAPINDACEAE & Pancovia-type & $\mathrm{x}$ & & & & & & & & & \\
\hline SAPINDACEAE & Placodiscus & $\mathrm{x}$ & & & & & $\mathrm{x}$ & & & & \\
\hline SAPINDACEAE & Sapindaceae undiff. & $\mathrm{x}$ & $\mathrm{x}$ & $\mathrm{x}$ & $\mathrm{x}$ & $\mathrm{x}$ & $\mathrm{x}$ & $\mathrm{x}$ & & & \\
\hline SAPOTACEAE & Sapotaceae undiff. & $\mathrm{x}$ & $\mathrm{x}$ & $\mathrm{x}$ & & & & & & & \\
\hline SOLANACEAE & Solanum-type & & & & & & & & & $\mathrm{x}$ & \\
\hline STERCULIACEAE & Cola cordifolia-type & & $\mathrm{x}$ & $\mathrm{x}$ & & & & & & & \\
\hline STERCULIACEAE & Mansonia altissima-type & & & $\mathrm{x}$ & & & & & & & \\
\hline STERCULIACEAE & Nesogordonia & & & $\mathrm{x}$ & & & & & & & \\
\hline STERCULIACEAE & Sterculiaceae undiff. & & $\mathrm{x}$ & $\mathrm{x}$ & $\mathrm{x}$ & $\mathrm{x}$ & & & & & \\
\hline STERCULIACEAE & Sterculia-type & & $\mathrm{x}$ & $\mathrm{x}$ & $\mathrm{x}$ & $\mathrm{x}$ & & & & & \\
\hline STERCULIACEAE & Triplochiton scleroxylon-type & & & $\mathrm{x}$ & & & & & & & \\
\hline THYMELAEACEAE & Thymelaeaceae undiff. & $\mathrm{x}$ & $\mathrm{x}$ & $\mathrm{x}$ & $\mathrm{x}$ & & $\mathrm{x}$ & & & & \\
\hline TILIACEAE & Grewia-type & $\mathrm{x}$ & $\mathrm{x}$ & $\mathrm{x}$ & $\mathrm{x}$ & $\mathrm{x}$ & $\mathrm{x}$ & & & & \\
\hline TILIACEAE & Tiliaceae undiff. & $\mathrm{x}$ & $\mathrm{x}$ & $\mathrm{x}$ & $\mathrm{x}$ & $\mathrm{x}$ & $\mathrm{x}$ & & & $\mathrm{x}$ & \\
\hline TILIACEAE & Triumfetta-type & & & & & & & & & $\mathrm{x}$ & \\
\hline ULMACEAE & Celtis & & $\mathrm{x}$ & $\mathrm{x}$ & & & & & & & \\
\hline ULMACEAE & Chaetacme aristata & & & $\mathrm{x}$ & & & & & & & \\
\hline ULMACEAE & Holoptelea grandis & & & $\mathrm{x}$ & & & & & & & \\
\hline ULMACEAE & Trema-type orientalis & $\mathrm{x}$ & $\mathrm{x}$ & $\mathrm{x}$ & $\mathrm{x}$ & $\mathrm{x}$ & & & & & \\
\hline ULMACEAE & Ulmaceae undiff. & $\mathrm{x}$ & $\mathrm{x}$ & $\mathrm{x}$ & $\mathrm{x}$ & $\mathrm{x}$ & & & & & \\
\hline URTICACEAE & Urticaceae undiff. & & & & & & $\mathrm{x}$ & & $\mathrm{x}$ & & \\
\hline VERBENACEAE & Vitex-type & & & $\mathrm{x}$ & $\mathrm{x}$ & $\mathrm{x}$ & & & & & \\
\hline VITACEAE & Cissus quadrangularis-type & & & & & & & & & $\mathrm{x}$ & \\
\hline VITACEAE & Vitaceae undiff. & & & & & & $\mathrm{x}$ & $\mathrm{x}$ & $\mathrm{x}$ & $\mathrm{x}$ & \\
\hline
\end{tabular}


Table 4. Atlantic Central African Biomes and their characteristic plant functional types (abbreviations for PFTs as in Table 2).

\begin{tabular}{lll}
\hline Codes & Biomes & Plant functional types \\
\hline TRFO & tropical rain forest & Te1, Te2, TLw, THw \\
TSFO & tropical seasonal forest & Te2, Tr1, TLw, THw \\
TDFO & tropical dry forest & Tr2, TLd, THd, g \\
SAV & savanna & Tr3, TLd, THd, g \\
\hline
\end{tabular}

evergreen), Te2 (dry tropical evergreen), $\operatorname{Tr} 1$ (wet tropical raingreen), $\operatorname{Tr} 2$ (dry tropical raingreen) and $\operatorname{Tr} 3$ (driest tropical raingreen) as defined by Jolly et al. (1998b) or Vincens et al. (2006) we have only included the tropical tree and shrub taxa. We have created four new PTFs, corresponding to tropical wet lianas (TLw), tropical dry lianas (TLd), tropical herbs from humid forest environments (THw) and from dry open environments (THd). As Peyron et al. (2000) and Vincens et al. (2006), grasses (Poaceae) represent a particular PFT (g). Thus, a total of 10 PFTs is used in this work (Table 2).

The 245 pollen taxa have been allocated to one or more of these PFTs (Table 3). When a taxa is assigned to more than one PFT, this is generally due to its low level of identification (family or genus). Thus, it can include several species with different biology (e.g. Rubiaceae, Danieilla, Parinari...) or it can comprise species that can adopt different life forms in different environments (e.g. Acacia a tree in savanna and a liana in forest....). The taxa-PFT allocation has been adapted to the study area and so shows many differences compared to the works of Jolly et al. (1998b), Peyron et al. (2000), Vincens et al. (2006) or Lézine et al. (2009). The corresponding plant life form and habitat of pollen taxa have been determined using West and Central African botanical literature (e.g. Flore du Congo Belge et du RuandaUrundi, 1948-1963; Flore du Congo, du Rwanda et du Burundi, 1967-1971; Flore d'Afrique Centrale (Zaïre, Rwanda, Burundi), 1972-2004; Hutchinson and Dalziel, 1954-1972; Flore du Gabon, 1961-2004; Flore du Cameroun, 19632001; Letouzey, 1968, 1985; Lebrun and Stork, 2003, 2006; Tchouto Mbatchou, 2004). Then, a final matrix involving the allocation of plant functional types to the 4 main biomes (TRFO [tropical rain forest], TSFO [tropical seasonal forest], TDFO [tropical dry forest] and SAVA [savanna]) occurring in Atlantic Central Africa has been created (Table 4).

\subsection{Potential succession stage reconstructions}

The same numerical procedure than the one used for potential biome reconstructions has been applied for the reconstruction of the succession stages of forest regeneration. The pollen data set comprises 250 taxa, including here Elaeis guineensis, Musanga, Anthocleista, Vismia guineensis and
Polyscias fulva, pioneer taxa which play an important role in the regeneration of the forest in its youngest stages.

The matrix taxa-PFTs comprises 14 PFTs including 13 new ones whose definition is based: (1) on the life form of the plants as for biome reconstructions and (2) on the place they occupy in the forest succession in function of their behaviour and growth strategies (savanna, regrowth, young secondary, old secondary and mature stages) (Richards, 1952; Descoings, 1969; Letouzey, 1968, 1985; Schell, 1976; Kahn, 1982; Catinot et al., 1983; White, 1983; White and Abernethy, 1996; Achoundong, 2000; Moutsamboté et al., 2000; Lebrun et Stork, 2003, 2006; Tchouto Mbatchou, 2004; de Namur, unpublished) (Table 5). Such succession status classification was already successfully applied for the aggregation of tropical tree species of the Sabah's lowland rain forests in Malaysia by Köhler et al. (2000) to suit for applications with process-based rain forest growth models. As above, the 250 pollen taxa have been allocated to one or more of these PFTs (Table 6).

Instead of "biomes" we have created dynamic "stages" of succession of forest regenerartion which are from the youngest to the oldest one:

- SAVA: corresponding to the grass herbaceous to semiwoody stage;

- TRFE: corresponding to the forest woody regrowth stage. In this stage the dominant shrubs and small trees, mainly heliophilous, such as Albizia, Anthocleista, Harungana, Tetrorchidium, Trema and Vernonia conferta are mixed with many coarse herbs (e.g. Zingiberaceae), soft woody shrubs and small climbers (e.g. Dioscorea).

- TYSF: characteristically this young secondary stage is dominated by the fast growing heliophilous Musanga cecropioides which is the most abundant and characteristic secondary forest tree in tropical Africa, associated with Myrianthus, Macaranga or Albizia for the most abundant trees. The herbaceous and shrubby layer is dense and lianas are abundant (e.g. Apocynaceae).

- TOSF: this old secondary stage is dominated by semiheliophilous species of moderately rapid growth. Characteristic species occurring in the canopy are: Alstonia boonei, Canarium, Ceiba pentandra, Zanthoxylum macrophylllum, Pycnanthus angolensis, Terminalia superba, Triplochiton scleroxylon....

- TMFO: This is the ultimate stage, or climacic mature stage, of forest regeneration. The floristic composition of this forest stage, the presence of shrub and herbaceous strata depends on the status of the forest: semi-deciduous or mixed semi-evergreen.

The final matrix involving the allocation of plant functional types to the 5 main succession stages is shown in Table 7 . 
Table 5. Allocation of the pollen taxa derived from all sites listed in Table 1 to the plant functional types used in dynamics reconstructions.

\begin{tabular}{|c|c|c|c|c|c|c|c|c|c|c|c|c|c|c|c|}
\hline Family & Taxon & Tma & Lma & Hma & Tosf & Tysf & Lsf & Hsf & Tpi & Lpi & Hpi & $\operatorname{Tr} 3$ & Lr3 & $\mathrm{Hr} 3$ & g \\
\hline ACANTHACEAE & Acanthaceae undiff. & $\mathrm{x}$ & $\mathrm{x}$ & $\mathrm{x}$ & $\mathrm{x}$ & $\mathrm{x}$ & $\mathrm{x}$ & $\mathrm{x}$ & $\mathrm{x}$ & $\mathrm{x}$ & $\mathrm{x}$ & & & $\mathrm{x}$ & \\
\hline ACANTHACEAE & Anisotes & $\mathrm{x}$ & & & & & & & & & & & & & \\
\hline ACANTHACEAE & Asystasia gangetica-type & & & $\mathrm{x}$ & & & & & & & $\mathrm{x}$ & & & & \\
\hline ACANTHACEAE & Mendoncia & & $\mathrm{x}$ & & & & & & & & & & & & \\
\hline ACANTHACEAE & Thomandersia & $\mathrm{x}$ & & & & & & & & & & & & & \\
\hline AMARANTHACEAE & Achyranthes-type aspera & & & & & & & & & & $\mathrm{x}$ & & & $\mathrm{x}$ & \\
\hline AMARANTHACEAE & Cyathula-type & & & $\mathrm{x}$ & & & & $\mathrm{x}$ & & & $\mathrm{x}$ & & & $\mathrm{x}$ & \\
\hline AMARANTHACEAE & Sericostachys-type scandens & & $\mathrm{x}$ & & & & $\mathrm{x}$ & & & $\mathrm{x}$ & & & & & \\
\hline AMARANTHACEAE/CHENOPODIACEAE & Amaranthaceae/Chenopodiaceae undiff. & & $\mathrm{x}$ & $\mathrm{x}$ & & & $\mathrm{x}$ & $\mathrm{x}$ & & $\mathrm{x}$ & $\mathrm{x}$ & & & $\mathrm{x}$ & \\
\hline ANACARDIACEAE & Anacardiaceae undiff. & $\mathrm{x}$ & $\mathrm{x}$ & & $\mathrm{x}$ & $\mathrm{x}$ & & & $\mathrm{x}$ & & & $\mathrm{x}$ & & & \\
\hline ANACARDIACEAE & Antrocaryon-type & $\mathrm{x}$ & & & $\mathrm{x}$ & & & & & & & & & & \\
\hline ANACARDIACEAE & Antrocaryon-type klaineanum & $\mathrm{x}$ & & & $\mathrm{x}$ & & & & & & & & & & \\
\hline ANACARDIACEAE & Fegimanra & & & & & & & & $\mathrm{x}$ & & & $\mathrm{x}$ & & & \\
\hline ANACARDIACEAE & Lannea-type & $\mathrm{x}$ & & & $\mathrm{x}$ & $\mathrm{x}$ & & & $\mathrm{x}$ & & & $\mathrm{x}$ & & & \\
\hline ANACARDIACEAE & Pseudospondias-type & $\mathrm{x}$ & & & & & & & & & & & & & \\
\hline ANACARDIACEAE & Sorindeia-type & $\mathrm{x}$ & & & & & & & & & & & & & \\
\hline ANACARDIACEAE & Trichoscypha-type & $\mathrm{x}$ & $\mathrm{x}$ & & & & & & & & & & & & \\
\hline ANISOPHYLLEACEAE & Anisophyllea & $\mathrm{x}$ & & & $\mathrm{x}$ & $\mathrm{x}$ & & & $\mathrm{x}$ & & & & & & \\
\hline ANISOPHYLLEACEAE & Anopyxis klaineana & $\mathrm{x}$ & & & $\mathrm{x}$ & & & & & & & & & & \\
\hline ANNONACEAE & Annonaceae undiff. & $\mathrm{x}$ & $\mathrm{x}$ & & $\mathrm{x}$ & $\mathrm{x}$ & $\mathrm{x}$ & & $\mathrm{x}$ & & & & & & \\
\hline APIACEAE & Apiaceae undiff. & & & & & & & & & & $\mathrm{x}$ & & & $\mathrm{x}$ & \\
\hline APOCYNACEAE & Alstonia-type & $\mathrm{x}$ & & & $\mathrm{x}$ & & & & & & & & & & \\
\hline APOCYNACEAE & Alstonia-type boonei & $\mathrm{x}$ & & & $\mathrm{x}$ & & & & & & & & & & \\
\hline APOCYNACEAE & Apocynaceae undiff. & $\mathrm{x}$ & $\mathrm{x}$ & & $\mathrm{x}$ & $\mathrm{x}$ & $\mathrm{x}$ & & $\mathrm{x}$ & $\mathrm{x}$ & & $\mathrm{x}$ & $\mathrm{x}$ & & \\
\hline APOCYNACEAE & Funtumia-type & $\mathrm{x}$ & & & $\mathrm{x}$ & $\mathrm{x}$ & & & & & & & & & \\
\hline APOCYNACEAE & Landolphia-type & & $\mathrm{x}$ & & & & $\mathrm{x}$ & & & $\mathrm{x}$ & & & $\mathrm{x}$ & & \\
\hline APOCYNACEAE & Oncinotis-type & & $\mathrm{x}$ & & & & $\mathrm{x}$ & & & $\mathrm{x}$ & & & & & \\
\hline APOCYNACEAE & Picralima-type nitida & $\mathrm{x}$ & & & & & & & & & & & & & \\
\hline APOCYNACEAE & Pleiocarpa & $\mathrm{x}$ & & & & & & & & & & & & & \\
\hline APOCYNACEAE & Rauvolfia & $\mathrm{x}$ & & & $\mathrm{x}$ & $\mathrm{x}$ & & & & & & & & & \\
\hline APOCYNACEAE & Tabernaemontana & $\mathrm{x}$ & & & $\mathrm{x}$ & $\mathrm{x}$ & & & $\mathrm{x}$ & & & & & & \\
\hline ARALIACEAE & Polyscias fulva-type & & & & $\mathrm{x}$ & $\mathrm{x}$ & & & $\mathrm{x}$ & & & & & & \\
\hline ASTERACEAE & Asteraceae undiff. & & & & & & & & & & & $\mathrm{x}$ & & $\mathrm{x}$ & \\
\hline ASTERACEAE & Vernonieae undiff. & & & & $\mathrm{x}$ & $\mathrm{x}$ & & & $\mathrm{x}$ & & $\mathrm{x}$ & $\mathrm{x}$ & & $\mathrm{x}$ & \\
\hline BALANITACEAE & Balanites & $\mathrm{x}$ & & & & & & & & & & & & & \\
\hline BEGONIACEAE & Begonia & & & $\mathrm{x}$ & & & & & & & & & & & \\
\hline BOMBACACEAE & Bombacaceae undiff. & $\mathrm{x}$ & & & $\mathrm{x}$ & & & & & & & & & & \\
\hline BOMBACACEAE & Ceiba pentandra & $\mathrm{x}$ & & & $\mathrm{x}$ & & & & & & & & & & \\
\hline BORAGINACEAE & Boraginaceae undiff. & $\mathrm{x}$ & & & $\mathrm{x}$ & $\mathrm{x}$ & & & $\mathrm{x}$ & & $\mathrm{x}$ & $\mathrm{x}$ & & $\mathrm{x}$ & \\
\hline BORAGINACEAE & Cordia platythyrsa-type & $\mathrm{x}$ & & & $\mathrm{x}$ & $\mathrm{x}$ & & & $\mathrm{x}$ & & & & & & \\
\hline BORAGINACEAE & Ehretia & $\mathrm{x}$ & & & $\mathrm{x}$ & $\mathrm{x}$ & & & & & & $\mathrm{x}$ & & & \\
\hline BORAGINACEAE & Heliotropium indicum-type & & & & & & & & & & $\mathrm{x}$ & & & $\mathrm{x}$ & \\
\hline BORAGINACEAE & Heliotropium steudneri-type & & & & & & & & & & & & & $\mathrm{x}$ & \\
\hline BURSERACEAE & Aucoumea klaineana & $\mathrm{x}$ & & & $\mathrm{x}$ & $\mathrm{x}$ & & & $\mathrm{x}$ & & & & & & \\
\hline BURSERACEAE & Burseraceae undiff. & $\mathrm{x}$ & & & $\mathrm{x}$ & & & & & & & $\mathrm{x}$ & & & \\
\hline BURSERACEAE & Canarium-type & $\mathrm{x}$ & & & $\mathrm{x}$ & & & & & & & & & & \\
\hline BURSERACEAE & Commiphora edulis-type & & & & & & & & & & & $\mathrm{x}$ & & & \\
\hline BURSERACEAE & Dacryodes-type & $\mathrm{x}$ & & & & & & & & & & & & & \\
\hline BURSERACEAE & Santiria-type & $\mathrm{x}$ & & & & & & & & & & & & & \\
\hline CAESALPINIACEAE & Anthonotha-type & $\mathrm{x}$ & & & & & & & & & & & & & \\
\hline CAESALPINIACEAE & Berlinia-type & $\mathrm{x}$ & & & & & & & & & & & & & \\
\hline CAESALPINIACEAE & Caesalpiniaceae undiff. & $\mathrm{x}$ & $\mathrm{x}$ & & $\mathrm{x}$ & $\mathrm{x}$ & & & $\mathrm{x}$ & & $\mathrm{x}$ & $\mathrm{x}$ & & $\mathrm{x}$ & \\
\hline CAESALPINIACEAE & Copaifera-type & $\mathrm{x}$ & & & & & & & & & & & & & \\
\hline CAESALPINIACEAE & Crudia-type gabonensis & $\mathrm{x}$ & & & & & & & & & & & & & \\
\hline
\end{tabular}


Table 5. Continued.

\begin{tabular}{|c|c|c|c|c|c|c|c|c|c|c|c|c|c|c|c|}
\hline Family & Taxon & Tma & Lma & Hma & Tosf & Tysf & Lsf & Hsf & Tpi & Lpi & Hpi & $\operatorname{Tr} 3$ & Lr3 & $\mathrm{Hr} 3$ & $\mathrm{~g}$ \\
\hline [2mm] CAESALPINIACEAE & Daniellia & $\mathrm{x}$ & & & $\mathrm{x}$ & $\mathrm{x}$ & & & & & & $\mathrm{x}$ & & & \\
\hline CAESALPINIACEAE & Detarium & $\mathrm{x}$ & & & & & & & & & & $\mathrm{x}$ & & & \\
\hline CAESALPINIACEAE & Dialium & $\mathrm{x}$ & & & & & & & & & & $\mathrm{x}$ & & & \\
\hline CAESALPINIACEAE & Dialium pachyphyllum-type & $\mathrm{x}$ & & & & & & & & & & & & & \\
\hline CAESALPINIACEAE & Distemonanthus benthamianus-type & $\mathrm{x}$ & & & $\mathrm{x}$ & & & & & & & & & & \\
\hline CAESALPINIACEAE & Duparquetia orchidacea & & & & & & & & $\mathrm{x}$ & & & $\mathrm{x}$ & & & \\
\hline CAESALPINIACEAE & Gilbertiodendron-type & $\mathrm{x}$ & & & & & & & & & & & & & \\
\hline CAESALPINIACEAE & Guibourtia & $\mathrm{x}$ & & & & & & & & & & & & & \\
\hline CAESALPINIACEAE & Guibourtia demeusei-type & $\mathrm{x}$ & & & & & & & & & & & & & \\
\hline CAESALPINIACEAE & Hylodendron gabunense & $\mathrm{x}$ & & & $\mathrm{x}$ & & & & & & & & & & \\
\hline CAESALPINIACEAE & Hymenostegia-type pellegrinii & $\mathrm{x}$ & & & & & & & & & & & & & \\
\hline CAESALPINIACEAE & Tessmannia & $\mathrm{x}$ & & & & & & & & & & & & & \\
\hline CAPPARIDACEAE & Capparidaceae undiff. & $\mathrm{x}$ & $\mathrm{x}$ & & $\mathrm{x}$ & $\mathrm{x}$ & $\mathrm{x}$ & $\mathrm{x}$ & & & $\mathrm{x}$ & $\mathrm{x}$ & $\mathrm{x}$ & $\mathrm{x}$ & \\
\hline CELASTRACEAE/HIPPOCRATEACEAE & Celastraceae/Hippocrateaceae undiff. & $\mathrm{x}$ & $\mathrm{x}$ & & $\mathrm{x}$ & $\mathrm{x}$ & $\mathrm{x}$ & & $\mathrm{x}$ & $\mathrm{x}$ & & $\mathrm{x}$ & $\mathrm{x}$ & & \\
\hline CHRYSOBALANACEAE & Chrysobalanus-type icaco & $\mathrm{x}$ & & & & & & & & & & & & & \\
\hline CHRYSOBALANACEAE & Maranthes-type & $\mathrm{x}$ & & & & & & & & & & & & & \\
\hline CHRYSOBALANACEAE & Parinari-type & $\mathrm{x}$ & & & & & & & & & & $\mathrm{x}$ & & & \\
\hline CLUSIACEAE & Allanblackia & $\mathrm{x}$ & & & & & & & & & & & & & \\
\hline CLUSIACEAE & Garcinia epunctata-type & $\mathrm{x}$ & & & & & & & & & & & & & \\
\hline CLUSIACEAE & Mammea africana-type & $\mathrm{x}$ & & & & & & & & & & & & & \\
\hline COMBRETACEAE & Combretaceae undiff. & $\mathrm{x}$ & $\mathrm{x}$ & & $\mathrm{x}$ & $\mathrm{x}$ & $\mathrm{x}$ & & $\mathrm{x}$ & $\mathrm{x}$ & & $\mathrm{x}$ & & & \\
\hline COMBRETACEAE & Terminalia-type & $\mathrm{x}$ & & & $\mathrm{x}$ & & & & & & & $\mathrm{x}$ & & & \\
\hline COMBRETACEAE/MELASTOMATACEAE & Combretaceae/Melastomataceae undiff. & $\mathrm{x}$ & $\mathrm{x}$ & $\mathrm{x}$ & $\mathrm{x}$ & $\mathrm{x}$ & $\mathrm{x}$ & $\mathrm{x}$ & $\mathrm{x}$ & $\mathrm{x}$ & $\mathrm{x}$ & $\mathrm{x}$ & & $\mathrm{x}$ & \\
\hline CONNARACEAE & Cnestis & $\mathrm{x}$ & $\mathrm{x}$ & & & $\mathrm{x}$ & $\mathrm{x}$ & & & $\mathrm{x}$ & & & & & \\
\hline CONVOLVULACEAE & Convolvulaceae undiff. & & $\mathrm{x}$ & $\mathrm{x}$ & & & & $\mathrm{x}$ & & & $\mathrm{x}$ & & & $\mathrm{x}$ & \\
\hline CONVOLVULACEAE & Evolvulus-type & & & & & & & & & & & & & $\mathrm{x}$ & \\
\hline CONVOLVULACEAE & Ipomoea-type & & & $\mathrm{x}$ & & & & $\mathrm{x}$ & & & $\mathrm{x}$ & & & $\mathrm{x}$ & \\
\hline CUCURBITACEAE & Coccinia & & & & & & & $\mathrm{x}$ & & & $\mathrm{x}$ & & & $\mathrm{x}$ & \\
\hline CUCURBITACEAE & Cucurbitaceae undiff. & & & $\mathrm{x}$ & & & & $\mathrm{x}$ & & & $\mathrm{x}$ & & & $\mathrm{x}$ & \\
\hline CUCURBITACEAE & Luffa-type & & & & & & & & & & $\mathrm{x}$ & & & $\mathrm{x}$ & \\
\hline DILLENIACEAE & Tetracera & $\mathrm{x}$ & $\mathrm{x}$ & & $\mathrm{x}$ & $\mathrm{x}$ & $\mathrm{x}$ & & $\mathrm{x}$ & $\mathrm{x}$ & & & & & \\
\hline DIOSCOREACEAE & Dioscorea & & & $\mathrm{x}$ & & & & $\mathrm{x}$ & & & $\mathrm{x}$ & & & $\mathrm{x}$ & \\
\hline DRACAENACEAE & Dracaena & $\mathrm{x}$ & & & $\mathrm{x}$ & $\mathrm{x}$ & & & $\mathrm{x}$ & & & $\mathrm{x}$ & & & \\
\hline EBENACEAE & Diospyros & $\mathrm{x}$ & & & & & & & & & & & & & \\
\hline EUPHORBIACEAE & Acalypha & $\mathrm{x}$ & & & $\mathrm{x}$ & $\mathrm{x}$ & & & $\mathrm{x}$ & & $\mathrm{x}$ & $\mathrm{x}$ & & $\mathrm{x}$ & \\
\hline EUPHORBIACEAE & Alchornea & $\mathrm{x}$ & & & $\mathrm{x}$ & $\mathrm{x}$ & & & $\mathrm{x}$ & & & & & & \\
\hline EUPHORBIACEAE & Anthostema-type & $\mathrm{x}$ & & & & & & & & & & & & & \\
\hline EUPHORBIACEAE & Antidesma-type & $\mathrm{x}$ & & & $\mathrm{x}$ & $\mathrm{x}$ & & & $\mathrm{x}$ & & & $\mathrm{x}$ & & & \\
\hline EUPHORBIACEAE & Bridelia ferruginea-type & $\mathrm{x}$ & & & $\mathrm{x}$ & $\mathrm{x}$ & & & $\mathrm{x}$ & & & $\mathrm{x}$ & & & \\
\hline EUPHORBIACEAE & Bridelia micrantha-type & $\mathrm{x}$ & & & $\mathrm{x}$ & $\mathrm{x}$ & & & $\mathrm{x}$ & & & $\mathrm{x}$ & & & \\
\hline EUPHORBIACEAE & Centroplacus glaucinus & $\mathrm{x}$ & & & $\mathrm{x}$ & & & & & & & & & & \\
\hline EUPHORBIACEAE & Cleistanthus-type polystachyus & $\mathrm{x}$ & & & & & & & & & & & & & \\
\hline EUPHORBIACEAE & Croton-type & $\mathrm{x}$ & & & $\mathrm{x}$ & $\mathrm{x}$ & & & $\mathrm{x}$ & & & & & & \\
\hline EUPHORBIACEAE & Cyathogyne & $\mathrm{x}$ & & $\mathrm{x}$ & & & & & & & & & & & \\
\hline EUPHORBIACEAE & Cyttaranthus congolensis & $\mathrm{x}$ & & & & & & & & & & & & & \\
\hline EUPHORBIACEAE & Discoglypremna caloneura & $\mathrm{x}$ & & & $\mathrm{x}$ & & & & & & & & & & \\
\hline EUPHORBIACEAE & Drypetes-type & $\mathrm{x}$ & & & & & & & & & & & & & \\
\hline EUPHORBIACEAE & Elaeophorbia-type & $\mathrm{x}$ & & & $\mathrm{x}$ & $\mathrm{x}$ & & & $\mathrm{x}$ & & & & & & \\
\hline EUPHORBIACEAE & Euphorbia-type & & & & & & & & & & $\mathrm{x}$ & & & $\mathrm{x}$ & \\
\hline EUPHORBIACEAE & Euphorbiaceae undiff. & $\mathrm{x}$ & $\mathrm{x}$ & $\mathrm{x}$ & $\mathrm{x}$ & $\mathrm{x}$ & & $\mathrm{x}$ & $\mathrm{x}$ & & $\mathrm{x}$ & $\mathrm{x}$ & & $\mathrm{x}$ & \\
\hline EUPHORBIACEAE & Klaineanthus gaboniae & $\mathrm{x}$ & & & & & & & & & & & & & \\
\hline EUPHORBIACEAE & Macaranga-type & $\mathrm{x}$ & & & $\mathrm{x}$ & $\mathrm{x}$ & & & $\mathrm{x}$ & & & & & & \\
\hline EUPHORBIACEAE & Mallotus-type oppositifolius & $\mathrm{x}$ & & & $\mathrm{x}$ & $\mathrm{x}$ & & & $\mathrm{x}$ & & & & & & \\
\hline
\end{tabular}


Table 5. Continued.

\begin{tabular}{|c|c|c|c|c|c|c|c|c|c|c|c|c|c|c|c|}
\hline Family & Taxon & Tma & Lma & Hma & Tosf & Tysf & Lsf & Hsf & Tpi & Lpi & Hpi & $\operatorname{Tr} 3$ & Lr3 & $\mathrm{Hr} 3$ & $\mathrm{~g}$ \\
\hline EUPHORBIACEAE & Margaritaria discoidea & $\mathrm{x}$ & & & $\mathrm{x}$ & $\mathrm{x}$ & & & $\mathrm{x}$ & & & & & & \\
\hline EUPHORBIACEAE & Martretia quadricornis & $\mathrm{x}$ & & & & & & & & & & & & & \\
\hline EUPHORBIACEAE & Phyllanthus-type & $\mathrm{x}$ & & $\mathrm{x}$ & $\mathrm{x}$ & $\mathrm{x}$ & & $\mathrm{x}$ & $\mathrm{x}$ & & $\mathrm{x}$ & $\mathrm{x}$ & & $\mathrm{x}$ & \\
\hline EUPHORBIACEAE & Plagiostyles-type africana & $\mathrm{x}$ & & & $\mathrm{x}$ & $\mathrm{x}$ & & & & & & & & & \\
\hline EUPHORBIACEAE & Tetrorchidium & $\mathrm{x}$ & & & $\mathrm{x}$ & $\mathrm{x}$ & & & $\mathrm{x}$ & & & & & & \\
\hline EUPHORBIACEAE & Uapaca & $\mathrm{x}$ & & & $\mathrm{x}$ & & & & & & & & & & \\
\hline EUPHORBIACEAE & Uapaca guineensis-type & $\mathrm{x}$ & & & $\mathrm{x}$ & & & & & & & & & & \\
\hline EUPHORBIACEAE & Uapaca heudelotii-type & $\mathrm{x}$ & & & & & & & & & & & & & \\
\hline FABACEAE & Aeschynomene baumii-type & & & & & & & & & & & $\mathrm{x}$ & & & \\
\hline FABACEAE & Baphia-type & $\mathrm{x}$ & & & $\mathrm{x}$ & $\mathrm{x}$ & & & $\mathrm{x}$ & & & & & & \\
\hline FABACEAE & Fabaceae undiff. & $\mathrm{x}$ & $\mathrm{x}$ & & $\mathrm{x}$ & $\mathrm{x}$ & $\mathrm{x}$ & $\mathrm{x}$ & $\mathrm{x}$ & $\mathrm{x}$ & $\mathrm{x}$ & $\mathrm{x}$ & $\mathrm{x}$ & $\mathrm{x}$ & \\
\hline FABACEAE & Indigofera & & & & & & & & & & $\mathrm{x}$ & & & $\mathrm{x}$ & \\
\hline FABACEAE & Pterocarpus-type & $\mathrm{x}$ & & & & & & & & & & $\mathrm{x}$ & & & \\
\hline FLACOURTIACEAE & Caloncoba-type & $\mathrm{x}$ & & & $\mathrm{x}$ & $\mathrm{x}$ & & & $\mathrm{x}$ & & & $\mathrm{x}$ & & & \\
\hline FLACOURTIACEAE & Camptostylus & $\mathrm{x}$ & & & & & & & & & & & & & \\
\hline FLACOURTIACEAE & Casearia & $\mathrm{x}$ & & & $\mathrm{x}$ & $\mathrm{x}$ & & & & & & $\mathrm{x}$ & & & \\
\hline FLACOURTIACEAE & Flacourtiaceae undiff. & $\mathrm{x}$ & & & $\mathrm{x}$ & $\mathrm{x}$ & & & $\mathrm{x}$ & & & $\mathrm{x}$ & & & \\
\hline FLACOURTIACEAE & Homalium & $\mathrm{x}$ & & & $\mathrm{x}$ & $\mathrm{x}$ & & & $\mathrm{x}$ & & & & & & \\
\hline FLACOURTIACEAE & Scottelia klaineana-type & $\mathrm{x}$ & & & $\mathrm{x}$ & & & & & & & & & & \\
\hline FLAGELLARIACEAE & Flagellaria & & & & & & & & & $\mathrm{x}$ & & & $\mathrm{x}$ & & \\
\hline HYMENOCARDIACEAE & Hymenocardia & $\mathrm{x}$ & & & & & & & $\mathrm{x}$ & & & $\mathrm{x}$ & & & \\
\hline HYMENOCARDIACEAE & Hymenocardia ulmoides-type & $\mathrm{x}$ & & & & & & & $\mathrm{x}$ & & & $\mathrm{x}$ & & & \\
\hline HYPERICACEAE & Harungana madagascariensis-type & & & & & $\mathrm{x}$ & & & $\mathrm{x}$ & & & $\mathrm{x}$ & & & \\
\hline HYPERICACEAE & Vismia guineensis & & & & & $\mathrm{x}$ & & & $\mathrm{x}$ & & & & & & \\
\hline ICACINACEAE & Icacinaceae undiff. & $\mathrm{x}$ & $\mathrm{x}$ & & $\mathrm{x}$ & $\mathrm{x}$ & $\mathrm{x}$ & & $\mathrm{x}$ & $\mathrm{x}$ & & & & & \\
\hline ICACINACEAE & Raphiostylis & $\mathrm{x}$ & $\mathrm{x}$ & & & & & & & & & & & & \\
\hline IRVINGIACEAE & Irvingia-type gabonensis & $\mathrm{x}$ & & & $\mathrm{x}$ & & & & & & & & & & \\
\hline LAMIACEAE & Hoslundia-type opposita & & & & & & & & $\mathrm{x}$ & & & $\mathrm{x}$ & & & \\
\hline LAMIACEAE & Lamiaceae undiff. & & & & & & & & $\mathrm{x}$ & & $\mathrm{x}$ & $\mathrm{x}$ & & $\mathrm{x}$ & \\
\hline LECYTHIDACEAE & Petersianthus-type macrocarpus & $\mathrm{x}$ & & & $\mathrm{x}$ & & & & & & & & & & \\
\hline LEGUMINOSAE & Leguminosae undiff. & $\mathrm{x}$ & $\mathrm{x}$ & & $\mathrm{x}$ & $\mathrm{x}$ & $\mathrm{x}$ & $\mathrm{x}$ & $\mathrm{x}$ & $\mathrm{x}$ & $\mathrm{x}$ & $\mathrm{x}$ & $\mathrm{x}$ & $\mathrm{x}$ & \\
\hline LOGANIACEAE & Anthocleista & & & & & $\mathrm{x}$ & & & $\mathrm{x}$ & & & $\mathrm{x}$ & & & \\
\hline LOGANIACEAE & Strychnos & $\mathrm{x}$ & $\mathrm{x}$ & & $\mathrm{x}$ & $\mathrm{x}$ & $\mathrm{x}$ & & $\mathrm{x}$ & & & $\mathrm{x}$ & & & \\
\hline LORANTHACEAE & Loranthaceae undiff. & $\mathrm{x}$ & & & & $\mathrm{x}$ & & & $\mathrm{x}$ & & & $\mathrm{x}$ & & & \\
\hline MALPIGHIACEAE & Acridocarpus & $\mathrm{x}$ & $\mathrm{x}$ & & $\mathrm{x}$ & $\mathrm{x}$ & $\mathrm{x}$ & & $\mathrm{x}$ & & & $\mathrm{x}$ & $\mathrm{x}$ & & \\
\hline MELASTOMATACEAE & Melastomataceae undiff. & $\mathrm{x}$ & & $\mathrm{x}$ & $\mathrm{x}$ & $\mathrm{x}$ & & $\mathrm{x}$ & $\mathrm{x}$ & & $\mathrm{x}$ & $\mathrm{x}$ & & $\mathrm{x}$ & \\
\hline MELIACEAE & Carapa-type procera & $\mathrm{x}$ & & & $\mathrm{x}$ & & & & & & & & & & \\
\hline MELIACEAE & Entandrophragma-type & $\mathrm{x}$ & & & $\mathrm{x}$ & & & & & & & & & & \\
\hline MELIACEAE & Khaya-type & $\mathrm{x}$ & & & $\mathrm{x}$ & & & & & & & & & & \\
\hline MELIACEAE & Meliaceae undiff. & $\mathrm{x}$ & & & $\mathrm{x}$ & $\mathrm{x}$ & & & $\mathrm{x}$ & & & & & & \\
\hline MELIACEAE & Trichilia-type & $\mathrm{x}$ & & & $\mathrm{x}$ & & & & & & & & & & \\
\hline MENISPERMACEAE & Menispermaceae undiff. & & $\mathrm{x}$ & & & & $\mathrm{x}$ & & & $\mathrm{x}$ & & & $\mathrm{x}$ & & \\
\hline MENISPERMACEAE & Tiliacora-type funifera & & $\mathrm{x}$ & & & & $\mathrm{x}$ & & & $\mathrm{x}$ & & & & & \\
\hline MIMOSACEAE & Acacia & & $\mathrm{x}$ & & & & $\mathrm{x}$ & & & $\mathrm{x}$ & & $\mathrm{x}$ & & & \\
\hline MIMOSACEAE & Albizia-type & & & & $\mathrm{x}$ & $\mathrm{x}$ & & & $\mathrm{x}$ & & & $\mathrm{x}$ & & & \\
\hline MIMOSACEAE & Calpocalyx-type & $\mathrm{x}$ & & & & & & & & & & & & & \\
\hline MIMOSACEAE & Calpocalyx-type letestui & $\mathrm{x}$ & & & & & & & & & & & & & \\
\hline MIMOSACEAE & Cylicodiscus-type gabunensis & $\mathrm{x}$ & & & & & & & & & & & & & \\
\hline MIMOSACEAE & Entada-type & & $\mathrm{x}$ & & & & $\mathrm{x}$ & & $\mathrm{x}$ & $\mathrm{x}$ & & $\mathrm{x}$ & $\mathrm{x}$ & & \\
\hline MIMOSACEAE & Mimosaceae undiff. & $\mathrm{x}$ & $\mathrm{x}$ & & $\mathrm{x}$ & $\mathrm{x}$ & $\mathrm{x}$ & & $\mathrm{x}$ & $\mathrm{x}$ & & $\mathrm{x}$ & $\mathrm{x}$ & & \\
\hline MIMOSACEAE & Parkia & $\mathrm{x}$ & & & & & & & & & & $\mathrm{x}$ & & & \\
\hline MIMOSACEAE & Pentaclethra macrophylla & $\mathrm{x}$ & & & $\mathrm{x}$ & $\mathrm{x}$ & & & & & & & & & \\
\hline MIMOSACEAE & Pentaclethra-type eetveldeana & $\mathrm{x}$ & & & $\mathrm{x}$ & $\mathrm{x}$ & & & & & & & & & \\
\hline MIMOSACEAE & Piptadeniastrum-type africanum & $\mathrm{x}$ & & & $\mathrm{x}$ & & & & & & & & & & \\
\hline MIMOSACEAE & Tetrapleura tetraptera-type & $\mathrm{x}$ & & & $\mathrm{x}$ & $\mathrm{x}$ & & & $\mathrm{x}$ & & & & & & \\
\hline MONOCOTYLEDONEAE & Monocotyledoneae undiff. & & & $\mathrm{x}$ & & & & $\mathrm{x}$ & & & $\mathrm{x}$ & & & $\mathrm{x}$ & \\
\hline MORACEAE & Antiaris-type toxicaria & $\mathrm{x}$ & & & & & & & & & & & & & \\
\hline
\end{tabular}


Table 5. Continued.

\begin{tabular}{|c|c|c|c|c|c|c|c|c|c|c|c|c|c|c|c|}
\hline Family & Taxon & Tma & Lma & Hma & Tosf & Tysf & Lsf & Hsf & Tpi & Lpi & Hpi & $\operatorname{Tr} 3$ & Lr3 & $\mathrm{Hr} 3$ & $\mathrm{~g}$ \\
\hline MORACEAE & Dorstenia-type & & & $\mathrm{x}$ & & & & $\mathrm{x}$ & & & & & & & \\
\hline MORACEAE & Ficus & $\mathrm{x}$ & $\mathrm{x}$ & & $\mathrm{x}$ & $\mathrm{x}$ & $\mathrm{x}$ & & $\mathrm{x}$ & & & $\mathrm{x}$ & & & \\
\hline MORACEAE & Milicia-type excelsa & $\mathrm{x}$ & & & $\mathrm{x}$ & & & & & & & & & & \\
\hline MORACEAE & Moraceae undiff. & $\mathrm{x}$ & $\mathrm{x}$ & & $\mathrm{x}$ & $\mathrm{x}$ & & & $\mathrm{x}$ & & & $\mathrm{x}$ & & & \\
\hline MORACEAE & Musanga-type & & & & & $\mathrm{x}$ & & & $\mathrm{x}$ & & & & & & \\
\hline MORACEAE & Myrianthus-type arboreus & $\mathrm{x}$ & & & $\mathrm{x}$ & $\mathrm{x}$ & & & $\mathrm{x}$ & & & & & & \\
\hline MORACEAE & Treculia & $\mathrm{x}$ & & & & & & & & & & & & & \\
\hline MORACEAE & Trilepisium-type madagascariensis & $\mathrm{x}$ & & & $\mathrm{x}$ & & & & & & & & & & \\
\hline MYRISTICACEAE & Coelocaryon & $\mathrm{x}$ & & & & & & & & & & & & & \\
\hline MYRISTICACEAE & Pycnanthus angolensis-type & $\mathrm{x}$ & & & $\mathrm{x}$ & & & & & & & & & & \\
\hline MYRISTICACEAE & Scyphocephalium & $\mathrm{x}$ & & & & & & & & & & & & & \\
\hline MYRISTICACEAE & Staudtia kamerunensis & $\mathrm{x}$ & & & & & & & & & & & & & \\
\hline MYRTACEAE & Syzygium-type & $\mathrm{x}$ & & & $\mathrm{x}$ & $\mathrm{x}$ & & & $\mathrm{x}$ & & & $\mathrm{x}$ & & & \\
\hline OCHNACEAE & Campylospermum & $\mathrm{x}$ & & & $\mathrm{x}$ & $\mathrm{x}$ & & & $\mathrm{x}$ & & & & & & \\
\hline OCHNACEAE & Lophira alata-type & $\mathrm{x}$ & & & $\mathrm{x}$ & $\mathrm{x}$ & & & $\mathrm{x}$ & & & & & & \\
\hline OLACACEAE & Coula edulis & $\mathrm{x}$ & & & & & & & & & & & & & \\
\hline OLACACEAE & Heisteria & $\mathrm{x}$ & & & & & & & & & & & & & \\
\hline OLACACEAE & Olax & $\mathrm{x}$ & & & & & & & & & & & & & \\
\hline OLACACEAE & Strombosia & $\mathrm{x}$ & & & & & & & & & & & & & \\
\hline OLACACEAE & Strombosia scheffleri-type & $\mathrm{x}$ & & & & & & & & & & & & & \\
\hline OLACACEAE & Strombosiopsis tetrandra & $\mathrm{x}$ & & & & & & & & & & & & & \\
\hline PALMAE & Borassus-type aethiopum & & & & & & & & & & & $\mathrm{x}$ & & & \\
\hline PALMAE & Elaeis guineensis & & & & $\mathrm{x}$ & $\mathrm{x}$ & & & $\mathrm{x}$ & & & & & & \\
\hline PALMAE & Podococcus barteri & $\mathrm{x}$ & & & & & & & & & & & & & \\
\hline PALMAE & Sclerosperma & $\mathrm{x}$ & & & & & & & & & & & & & \\
\hline PANDACEAE & Microdesmis & $\mathrm{x}$ & & & $\mathrm{x}$ & $\mathrm{x}$ & & & $\mathrm{x}$ & & & & & & \\
\hline POACEAE & Poaceae undiff. & & & & & & & & & & & & & & $\mathrm{x}$ \\
\hline RANUNCULACEAE & Clematis-type & & & & & & & & & $\mathrm{x}$ & & & $\mathrm{x}$ & & \\
\hline RHAMNACEAE & Rhamnaceae undiff. & $\mathrm{x}$ & $\mathrm{x}$ & & $\mathrm{x}$ & $\mathrm{x}$ & $\mathrm{x}$ & & $\mathrm{x}$ & $\mathrm{x}$ & & $\mathrm{x}$ & & & \\
\hline RUBIACEAE & Aidia-type & $\mathrm{x}$ & & & & & & & & & & & & & \\
\hline RUBIACEAE & Aidia-type micrantha & $\mathrm{x}$ & & & & & & & & & & & & & \\
\hline RUBIACEAE & Crossopteryx febrifuga & & & & & & & & $\mathrm{x}$ & & & $\mathrm{x}$ & & & \\
\hline RUBIACEAE & Hallea-type & $\mathrm{x}$ & & & & & & & & & & & & & \\
\hline RUBIACEAE & Hallea-type rubrostipulata & $\mathrm{x}$ & & & & & & & & & & & & & \\
\hline RUBIACEAE & Hymenodictyon-type floribundum & & & & & & & & & & & $\mathrm{x}$ & & & \\
\hline RUBIACEAE & Keetia-type gueinzii & $\mathrm{x}$ & $\mathrm{x}$ & & & & & & & & & $\mathrm{x}$ & $\mathrm{x}$ & & \\
\hline RUBIACEAE & Macrosphyra-type & & & & & & & & & & & $\mathrm{x}$ & $\mathrm{x}$ & & \\
\hline RUBIACEAE & Morinda & $\mathrm{x}$ & & & & & & & & & & $\mathrm{x}$ & & & \\
\hline RUBIACEAE & Nauclea-type & $\mathrm{x}$ & & & $\mathrm{x}$ & $\mathrm{x}$ & & & $\mathrm{x}$ & & & $\mathrm{x}$ & & & \\
\hline RUBIACEAE & Oldenlandia-type & & & & & & & & & & $\mathrm{x}$ & & & $\mathrm{x}$ & \\
\hline RUBIACEAE & Oligocodon-type cuniliffeae & & $\mathrm{x}$ & & & & & & & & & & & & \\
\hline RUBIACEAE & Pausinystalia-type macroceras & $\mathrm{x}$ & & & & & & & & & & & & & \\
\hline RUBIACEAE & Psychotria & $\mathrm{x}$ & & & $\mathrm{x}$ & $\mathrm{x}$ & & & $\mathrm{x}$ & & & & & & \\
\hline RUBIACEAE & Psydrax-type schimperiana & $\mathrm{x}$ & & & & & & & & & & & & & \\
\hline RUBIACEAE & Psydrax-type subcordata & $\mathrm{x}$ & & & & & & & & & & & & & \\
\hline RUBIACEAE & Rubiaceae undiff. & $\mathrm{x}$ & $\mathrm{x}$ & $\mathrm{x}$ & $\mathrm{x}$ & $\mathrm{x}$ & $\mathrm{x}$ & $\mathrm{x}$ & $\mathrm{x}$ & $\mathrm{x}$ & $\mathrm{x}$ & $\mathrm{x}$ & $\mathrm{x}$ & $\mathrm{x}$ & \\
\hline RUBIACEAE & Sherbournia bignoniiflora-type & & $\mathrm{x}$ & & & & & & & & & & & & \\
\hline RUBIACEAE & Spermacoce-type & & & & & & & & & & & & & $\mathrm{x}$ & \\
\hline RUBIACEAE & Uncaria-type africana & & $\mathrm{x}$ & & & & & & & & & & & & \\
\hline RUTACEAE & Rutaceae undiff. & $\mathrm{x}$ & $\mathrm{x}$ & & $\mathrm{x}$ & $\mathrm{x}$ & $\mathrm{x}$ & & $\mathrm{x}$ & & & & & & \\
\hline RUTACEAE & Vepris-type & $\mathrm{x}$ & & & & & & & & & & & & & \\
\hline RUTACEAE & Zanthoxylum-type & $\mathrm{x}$ & $\mathrm{x}$ & & $\mathrm{x}$ & $\mathrm{x}$ & $\mathrm{x}$ & & $\mathrm{x}$ & & & & & & \\
\hline
\end{tabular}


Table 5. Continued.

\begin{tabular}{|c|c|c|c|c|c|c|c|c|c|c|c|c|c|c|c|}
\hline Family & Taxon & Tma & Lma & Hma & Tosf & Tysf & Lsf & Hsf & Tpi & Lpi & Hрi & Tr3 & Lr3 & $\mathrm{Hr} 3$ & $\mathrm{~g}$ \\
\hline SAPINDACEAE & Allophylus & $\mathrm{x}$ & $\mathrm{x}$ & & $\mathrm{x}$ & $\mathrm{x}$ & $\mathrm{x}$ & & $\mathrm{x}$ & & & $\mathrm{x}$ & & & \\
\hline SAPINDACEAE & Aphania-type senegalensis & $\mathrm{x}$ & & & & & & & & & & & & & \\
\hline SAPINDACEAE & Blighia & $\mathrm{x}$ & & & & & & & & & & $\mathrm{x}$ & & & \\
\hline SAPINDACEAE & Cardiospermum & & $\mathrm{x}$ & & & & $\mathrm{x}$ & & & $\mathrm{x}$ & & & $\mathrm{x}$ & & \\
\hline SAPINDACEAE & Chytranthus-type & $\mathrm{x}$ & & & & & & & & & & & & & \\
\hline SAPINDACEAE & Dodonaea & & & & & & & & $\mathrm{x}$ & & & $\mathrm{x}$ & & & \\
\hline SAPINDACEAE & Eriocoelum & $\mathrm{x}$ & & & & & & & & & & & & & \\
\hline SAPINDACEAE & Ganophyllum-type giganteum & $\mathrm{x}$ & & & & & & & & & & & & & \\
\hline SAPINDACEAE & Laccodiscus & $\mathrm{x}$ & & & & & & & & & & & & & \\
\hline SAPINDACEAE & Lecaniodiscus-type & $\mathrm{x}$ & & & & & & & & & & & & & \\
\hline SAPINDACEAE & Pancovia-type & $\mathrm{x}$ & & & & & & & & & & & & & \\
\hline SAPINDACEAE & Placodiscus & $\mathrm{x}$ & $\mathrm{x}$ & & & $\mathrm{x}$ & & & & & & & & & \\
\hline SAPINDACEAE & Sapindaceae undiff. & $\mathrm{x}$ & $\mathrm{x}$ & & $\mathrm{x}$ & $\mathrm{x}$ & $\mathrm{x}$ & & $\mathrm{x}$ & $\mathrm{x}$ & & $\mathrm{x}$ & $\mathrm{x}$ & & \\
\hline SAPOTACEAE & Sapotaceae undiff. & $\mathrm{x}$ & & & & & & & & & & & & & \\
\hline SOLANACEAE & Solanum-type & & & & & & & & & & & & & $\mathrm{x}$ & \\
\hline STERCULIACEAE & Cola cordifolia-type & $\mathrm{x}$ & & & & & & & & & & & & & \\
\hline STERCULIACEAE & Mansonia altissima-type & $\mathrm{x}$ & & & & & & & & & & & & & \\
\hline STERCULIACEAE & Nesogordonia & $\mathrm{x}$ & & & & & & & & & & & & & \\
\hline STERCULIACEAE & Sterculiaceae undiff. & $\mathrm{x}$ & & & $\mathrm{x}$ & $\mathrm{x}$ & & & $\mathrm{x}$ & & & $\mathrm{x}$ & & & \\
\hline STERCULIACEAE & Sterculia-type & $\mathrm{x}$ & & & $\mathrm{x}$ & $\mathrm{x}$ & & & $\mathrm{x}$ & & & $\mathrm{x}$ & & & \\
\hline STERCULIACEAE & Triplochiton scleroxylon-type & $\mathrm{x}$ & & & $\mathrm{x}$ & & & & & & & & & & \\
\hline THYMELAEACEAE & Thymelaeaceae undiff. & $\mathrm{x}$ & $\mathrm{x}$ & & $\mathrm{x}$ & $\mathrm{x}$ & & & $\mathrm{x}$ & $\mathrm{x}$ & & $\mathrm{x}$ & & & \\
\hline TILIACEAE & Grewia-type & $\mathrm{x}$ & $\mathrm{x}$ & & $\mathrm{x}$ & $\mathrm{x}$ & & & $\mathrm{x}$ & & & $\mathrm{x}$ & & & \\
\hline TILIACEAE & Tiliaceae undiff. & $\mathrm{x}$ & $\mathrm{x}$ & & $\mathrm{x}$ & $\mathrm{x}$ & $\mathrm{x}$ & & $\mathrm{x}$ & & & $\mathrm{x}$ & & $\mathrm{x}$ & \\
\hline TILIACEAE & Triumfetta-type & & & & & & & & & & $\mathrm{x}$ & & & $\mathrm{x}$ & \\
\hline ULMACEAE & Celtis & $\mathrm{x}$ & & & $\mathrm{x}$ & $\mathrm{x}$ & & & & & & & & & \\
\hline ULMACEAE & Chaetacme aristata & $\mathrm{x}$ & & & $\mathrm{x}$ & $\mathrm{x}$ & & & $\mathrm{x}$ & & & & & & \\
\hline ULMACEAE & Holoptelea grandis & $\mathrm{x}$ & & & $\mathrm{x}$ & & & & & & & & & & \\
\hline ULMACEAE & Trema-type orientalis & $\mathrm{x}$ & & & $\mathrm{x}$ & $\mathrm{x}$ & & & $\mathrm{x}$ & & & $\mathrm{x}$ & & & \\
\hline ULMACEAE & Ulmaceae undiff. & $\mathrm{x}$ & & & $\mathrm{x}$ & $\mathrm{x}$ & & & $\mathrm{x}$ & & & $\mathrm{x}$ & & & \\
\hline URTICACEAE & Urticaceae undiff. & & $\mathrm{x}$ & $\mathrm{x}$ & & & $\mathrm{x}$ & $\mathrm{x}$ & & $\mathrm{x}$ & $\mathrm{x}$ & & & & \\
\hline VERBENACEAE & Vitex-type & $\mathrm{x}$ & & & $\mathrm{x}$ & $\mathrm{x}$ & & & $\mathrm{x}$ & & & $\mathrm{x}$ & & & \\
\hline VITACEAE & Cissus quadrangularis-type & & & & & & & & & & $\mathrm{x}$ & & & $\mathrm{x}$ & \\
\hline VITACEAE & Vitaceae undiff. & & $\mathrm{x}$ & & & & $\mathrm{x}$ & $\mathrm{x}$ & & $\mathrm{x}$ & $\mathrm{x}$ & & $\mathrm{x}$ & $\mathrm{x}$ & \\
\hline
\end{tabular}

\section{The results}

\subsection{Reconstruction of the Atlantic Central Africa Biomes}

\subsubsection{Comparison between reconstructed biomes and vegetation at each sampled site}

The results of the comparison (Table 8 and for detailed results refer to Table 1) show that among the 199 pollen sites considered in this study, 87 are correctly reconstructed as a potential biome Tropical Rain Forest (TRFO). They correspond to 74 sites of rain forest from Gabon and to 13 sites from the Cameroon littoral rain forest (8) and from the Dja forest area at the wet/dry rain forest transition (5). For the 43 other sites, all originating in the Mayombe forest massif in southern Congo, a potential TRFO biome is reconstructed. This result arises the problem of the status of this forest in
Atlantic Central Africa mapped by White (1983) as GuineoCongolian dry rain forest (Fig. 1), i.e. such as the Letouzey's semi-deciduous forest of southern Cameroon, north of $4^{\circ} \mathrm{N}$ (see specific discussion below, in Sect. 6.1.).

A potential biome Tropical Seasonal Forest (TSFO) is reconstructed at 49 sites. Among them, 45 sites from Cameroon, located in dry rain forest and 3 in the Dja forest area at the wet/dry rain forest transition are correctly reconstructed. Three other sites inside the wet rain forest of Gabon (2) and Cameroon (1) are clearly incorrectly reconstructed as potential TSFO biome such as one savanna in Cameroon.

All the 20 remaining sites are reconstructed as a potential biome Savanna (SAVA). Among them, 19 are really savanna, and one is from a forest regrowth, but largely disturbed by Man for cultivation, leading to high frequencies of Poaceae in the pollen spectra. 
Table 6. Atlantic Central African succession stages and their characteristic plant functional types (abbreviations for PFTs as in Table 4).

\begin{tabular}{lll}
\hline Codes & Stages & Plant functional types \\
\hline TMFO & tropical mature forest & Tma, Lma, Hma \\
TOSF & tropical old secondary forest & Tosf, Lsf, Hsf \\
TYSF & tropical young secondary forest & Tysf, Lsf, Hsf \\
TFRE & tropical forest regrowth & Tpi, Lpi, Hpi \\
SAVA & savanna & Tr3,Lr3, Hr3, g
\end{tabular}

\subsubsection{Comparison between reconstructed biomes and White's Central Atlantic vegetation types}

The results of the comparison are given in Fig. 1a and Table 9. Among the 199 sites, 65 sites from the wetter types of rain forest (i.e. Cameroon coastal evergreen forest and the Gabon mixed moist semi-evergreen rain forest) (vegetation type 1a, White, 1983) are correctly reconstructed as potential TRFO biome.

In the Dja area in southern Cameroon, where occurs the transition between the mixed moist semi-evergreen forest (type 1a) and the dry rain forest (type 2) (indicated in Table 9 as $1 \mathrm{a} / 2$ transition) the 9 sites can be considered as correctly reconstructed with 5 sites as potential TRFO biome and 4 sites as potential TSFO biome.

Inside the White's dry rain forest (type 2), only 8 Cameroon sites are correctly reconstructed as potential TSFO biome. One site is reconstructed as a SAVA but, corresponding locally to an enclosed savanna inside the forest, this reconstruction can be considered as correct. The 43 remaining sites are all the forest sites from the Congolese Mayombe massif which are reconstructed as potential TRFO biome and not as potential TSFO biome as it could be expected according to White's vegetation map.

The last White's vegetation type occurring in Central Africa (of drier type in southern Cameroon, and wetter type in central and southern Gabon) - mainly developed at the border of the forest massif - is the mosaic of rain forest and secondary grassland (type 11a). Inside this vegetation type, 17 Gabon sites are reconstructed as TRFO biome (Lopé area), 34 Cameroon sites as TSFO biome (Kandara area) and 19 Gabon and Cameroon sites as SAVA biome (Lopé area and southern Gabon, Kandara area) indicating well the mosaic character of the vegetation. For each site (total of 70), when the potential biome proposed is compared to the local vegetation as defined in the field (Table 1), it appears that all sites are well reconstructed. This shows the importance to have a minimum of botanical information at each sampling site.

\subsection{Reconstruction in terms of forest dynamics}

In a first step, we have considered all the succession stages which can be observed in a dynamics of reconstruction of the forest, from the younger herbaceous stage (SAVA) to the Tropical Mature FOrest stage (TMFO), including successively the Tropical Forest REgrowth (TFRE), the Tropical Young Secondary Forest (TYSF) then the Tropical Old Secondary Forest (TOSF). The results of the comparison between potential reconstructed stages and local vegetation show that only 3 stages can be considered as correctly reconstructed, with a number of correct assignments exceeding the number of incorrect ones (Table 10a and for details at each site refer to Table 1). These are the TMFO (148 sites), TOSF (6 sites) and SAVA (19 sites) stages. The youngest stages of arboreal recolonisation are poorly (TYSF) or totally uncorrected (TFRE) reconstructed.

According to these results we have re-arranged these stages into only 3 main stages: TMFO, TSFE (Tropical Secondary Forest) grouping all secondary succession stages, and SAVA (Table 10b and Fig. 1b). In this way, the potential stages correctly reconstructed are of $97.4 \%$ (148 sites), $66.6 \%$ (18 sites) and 95\% (21) of confidence, respectively.

\section{Discussion}

Our reconstructions in terms of biomes or succession stages of forest regeneration arise some questions concerning (1) the status of the Congolese Mayombe forest inside the Guineo-Congolian forest massif and (2) the boundary features between two biomes or two succession stages.

\subsection{The status of the Congolese Mayombe forest}

The potential biomes reconstructed at all the sites from the Congolese Mayombe forest show discrepancies compared with the White's Central Atlantic vegetation type locations. Indeed, all these sites are located in the dry rain forest (type 2) and are reconstructed as Tropical Rain Forest biome (TRFO) such as the Gabon forested sites and not as Tropical seasonal Forest biome (TSFO) as it could be expected (Table 1).

As in this work, White's vegetation map, based on a combination of physiognomic and floristic factors, is classically used by African palynologists to localise modern samples or fossil pollen sedimentary sequences (e.g. Lézine and Edorh, 1991; Vincens et al., 2006; Lézine et al., 2009). Sometimes it has been locally modified and complemented according to regional botanical and ecological data such as by Maley (1990), Maley and Elenga (1993) or more recently in Giresse et al. (2008) for Central Africa. But these authors have always conserved the status of semi-deciduous forest or dry rain forest as defined by White (1983) for the Mayombe forest in spite of detailed contradictory botanical field works in this area (e.g. Dowsett-Lemaire, 1991; Hecketsweiler and 
Table 7. Numerical comparison between pollen-derived (p) and observed (o) biomes at each sampled site.

\begin{tabular}{llllll}
\hline & TRFO (p) & TSFO (p) & TDFO (p) & SAVA (p) & $\begin{array}{l}\text { Potential biomes } \\
\text { correctly reconstructed (\%) }\end{array}$ \\
\hline TRFO (o) & $87+43$ & 3 & 0 & 0 & 97.7 \\
TSFO (o) & 0 & 45 & 0 & 1 & 97.8 \\
TDFO (o) & 0 & 0 & 0 & 0 & \\
SAVA (o) & 0 & 1 & 0 & 19 & 95 \\
\hline
\end{tabular}

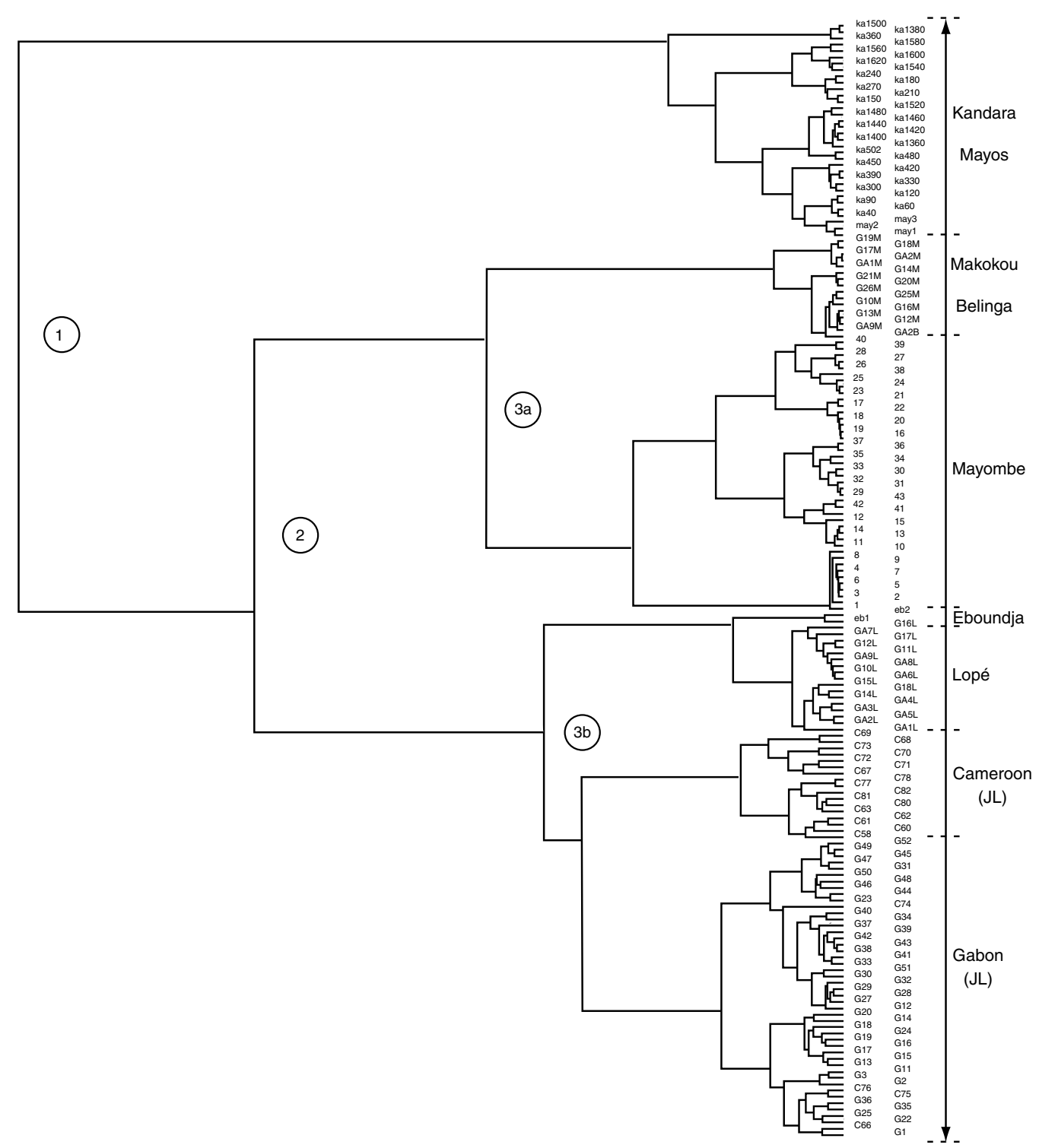

Fig. 2. Cluster analysis of the 176 modern pollen spectra from Atlantic Central African forest (JL: Lebamba et al., 2009). 
Table 8. Numerical comparison between pollen-derived biomes (p) and the main Atlantic Central African vegetation types defined by White (1983) (White's vegetation types: 1a - wetter types of Guineo-Congolian rain forest; 2 - drier types of Guineo-Congolian rain forest; 11a-mosaic of rain forest and secondary grassland).

\begin{tabular}{llll}
\hline & TRFO $(\mathrm{p})$ & TSFO $(\mathrm{p})$ & SAVA $(\mathrm{p})$ \\
\hline $1 \mathrm{a}$ & 65 & 3 & 0 \\
$1 \mathrm{a} / 2$ transition & 5 & 4 & 0 \\
2 & 43 (Congo) & 8 & 1 \\
$11 \mathrm{a}$ & 17 & 34 & 19 \\
\hline
\end{tabular}

Table 9. Plant functional types proposed for the Atlantic Central African areas under investigation for dynamic reconstructions.

\begin{tabular}{ll}
\hline Codes & Plant functional types \\
\hline Tma & tropical mature forest trees and shrubs \\
Lma & tropical mature forest lianas \\
Hma & tropical mature forest herbs \\
Tosf & tropical old secondary forest trees and shrubs \\
Tysf & tropical young secondary forest trees and shrubs \\
Lsf & tropical secondary forest lianas \\
Hsf & tropical secondary forest herbs \\
Tpi & tropical forest pioneer trees and shrubs (regrowth) \\
Lpi & tropical forest pioneer lianas (regrowth) \\
Hpi & tropical forest pioneer herbs (regrowth) \\
Tr3 & tropical savanna trees and shrubs \\
Lr3 & tropical savanna lianas \\
Hr3 & tropical savanna herbs \\
g & grasses \\
\hline
\end{tabular}

Mokoko Ikonga, 1991; Doumenge, 1992; de Namur, unpublished). As observed by Dowsett-Lemaire (1991) the striking feature of the Mayombe forest is the importance of the Caesalpiniaceae, locally dominant in the canopy. Among the dominant and most widespread emergent are found two families: the Irvingiaceae, and the Myristicaceae. Other very large and frequent trees belong to the Burseraceae, Combretaceae, Mimosaceae and Sapotaceae. The canopy in the Mayombe is also characterized by a significant proportion of briefly deciduous species; several species, however, are found essentially in secondary situations along roads and in abandoned farmlands and their importance must have in- creased with the spread of human disturbances. The epiphytic flora is especially rich in the central Mayombe between 400 and $500 \mathrm{~m}$ altitude. According to its floristic composition, Dowsett-Lemaire (1991) considers that the Mayombe forest is a rather complex assemblage of two main types of forest: the Atlantic coastal evergreen rain forest and the mixed moist semi-evergreen rain forest, and so, it must be classified as Guineo-Congolian wet rain forest. Such a conclusion seems to be confirmed by Doumenge (1992) who considers that the Mayombe forest has many affinities with the Biafran forest rich in Caesalpiniaceae described by Letouzey (1985) in Cameroon, extending southward in Central Gabon as evergreen forest (Nicolas, 1977; Caballé, 1978). Doumenge (1992) estimates that the Mayombe forest on well-drained soils is of dense wet semi-evergreen type. Before these works, Descoings (1969) mapped the Mayombe forest as equatorial rain forest, of the same type than those that occur in northern Congo on well-drained soils and Sita (1989) classified it as dense mixed wet semi-evergreen forest. Moreover, on the Central African vegetation map produced by de Namur (1990) the Mayombe massif appears as of transitional type between an evergreen type to a semideciduous one, as are mapped also forests from the northern Gabon (including the Makokou area) and from the southern Cameroon (including the Dja area).

In the light of these botanical information, we have analysed part of our pollen data set - i.e. considering only forest sites (176 and 272 taxa) - using hierarchical cluster analysis (Ward, 1963) and correspondence analysis (CA) (Benzécri, 1973) to identify the possible affinities between pollen assemblages from Congo and those from Gabon and Cameroon.

The dendrogram of the cluster analysis (Fig. 2) shows, at a first level of division (1), a clear differentiation between the Cameroon pollen spectra of Kandara and Mayos located in the northern dry peripheral rain forest (or semi-deciduous forest) and all the others. The next division (2) separates the spectra from Makokou and Belinga in Gabon and from Mayombe (Congo) from the others. At the third level of division (3a) spectra from Makokou and Belinga are well differentiated from those from the Mayombe and (3b) spectra from the Lopé (Gabon) are well separated from the other Cameroon and Gabon spectra. The Correspondence analysis (Fig. 3) displays the same features than the cluster analysis showing along the first axis a clear separation of pollen spectra from the dry peripheral rain forest of Cameroon (Kandara and Mayos) from the others, and along the axis 2 better affinities between the Congolese Mayombe spectra and the Makokou and Belinga ones than with all the others.

These results show that the Mayombe forest, though under lower mean annual rainfall $(1400 \mathrm{~mm} /$ year $)$ and a longer dry season of 4 months, has more floristic affinities with the wet inland evergreen semi-evergreen rain forest occurring in Gabon (mean of $1600 \mathrm{~mm} / \mathrm{year}$, a dry season of 3 months) as previously proposed by Doumenge (1992) and 
Table 10. Numerical comparison between pollen-derived (p) and observed (o) succession stages at each sampled site.

\begin{tabular}{lllllll}
\hline (a) & TMFO (p) & TOSF (p) & TYSF (p) & TFRE (p) & SAVA (p) & $\begin{array}{l}\text { Potential stages } \\
\text { correctly reconstructed (\%) }\end{array}$ \\
\hline TMFO (o) & $\mathbf{1 4 8}$ & 4 & 0 & 0 & 0 & 97.4 \\
TOSF (o) & 3 & $\mathbf{6}$ & 0 & 0 & 0 & 66.6 \\
TYSF (o) & 3 & 9 & $\mathbf{1}$ & 0 & 0 & 7.6 \\
TFRE (o) & 1 & 1 & 1 & $\mathbf{0}$ & 2 & 0 \\
SAVA (o) & 0 & 1 & 0 & 0 & $\mathbf{1 9}$ & 95 \\
& & & & & \\
\hline (b) & TMFO (p) & TSFE (p) & SAVA (p) & Potential stages correctly reconstructed (\%) \\
\hline TMFO (o) & $\mathbf{1 4 8}$ & 4 & 0 & & 97.4 \\
TSFE (o) (TOSF + TYSF + TFRE) & 7 & $\mathbf{1 8}$ & 2 & & 66.6 \\
SAVA (o) & 0 & 1 & $\mathbf{1 9}$ & & \\
\hline
\end{tabular}

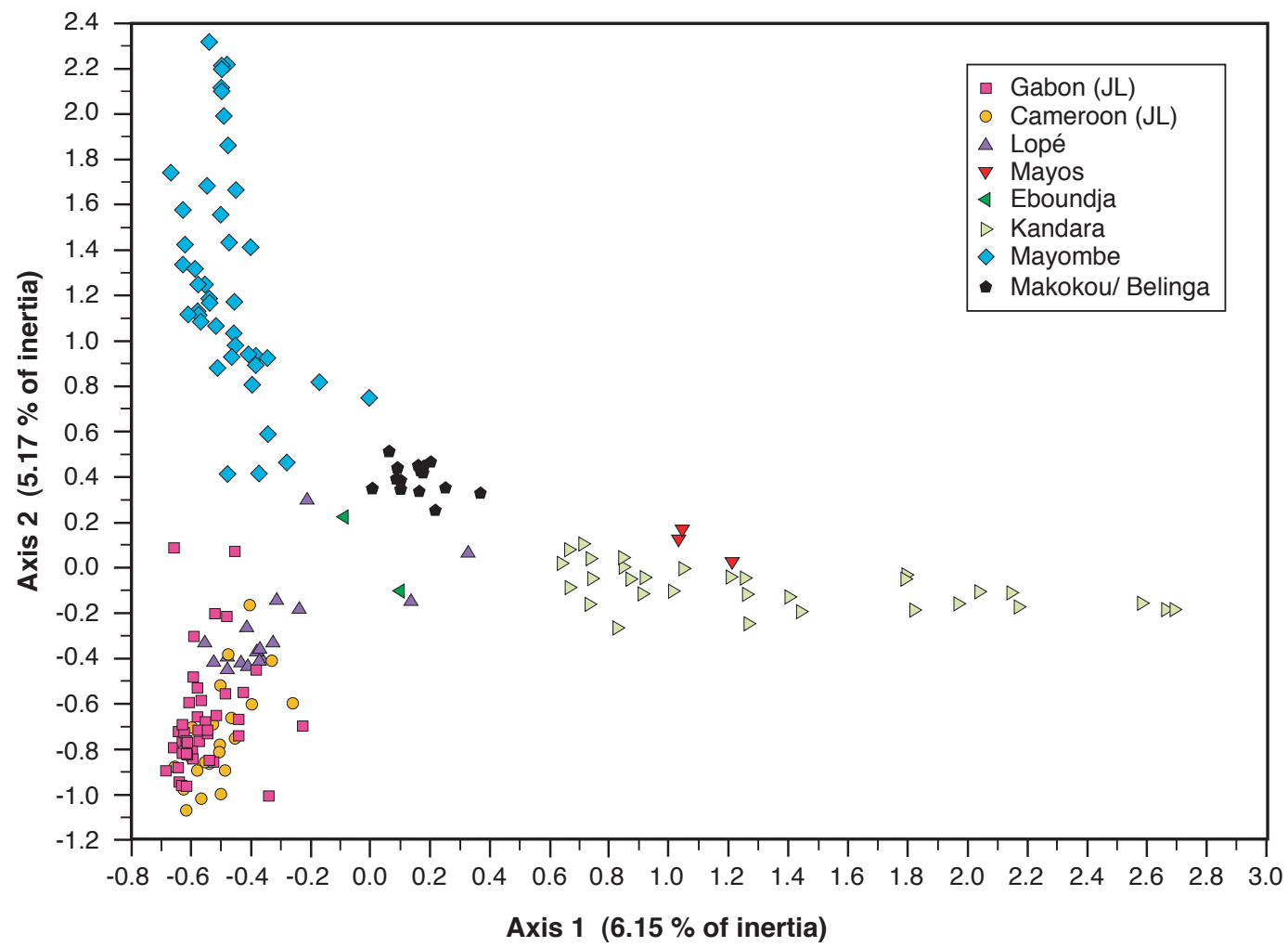

Fig. 3. Correspondence analysis with respect to CA axes 1 and 2 of the 176 modern pollen spectra from Atlantic Central African forest (JL: Lebamba et al., 2009).

mapped by de Namur (1990), than with the dry Cameroon semi-deciduous rain forest $(1600 \mathrm{~mm} / \mathrm{year}$, a dry season of 3 months) as mapped by White (1983). This would confirm the hypothesis expressed by Lebamba et al. (2009) suggesting the importance of the role played by the cloud cover and the relative atmospheric humidity during the dry season in the floristic composition of forests north and south of the meteorological equator, rather than the annual rainfall amount and/or the length of the dry season. High values of these two climatic parameters, linked to monsoon influences from 
the Gulf of Guinea in Congo and Gabon, are measured during June, July and August versus low values in Cameroon linked to influences of continental trade winds (Harmattan) during December, January and February (Nicholson, 2000; FAO Web LocClim, 2008).

\subsection{The boundaries between biomes or succession stages}

While the boundary between grassland and rain forest is clearly identified in the field and on aerial or satellite photographs, by their floristic composition, structure and physiognomy, as well as in pollen assemblages by the abundance of the Poaceae versus arboreal taxa, inside the forest massif the limit between the wet and the dry rain forest, and between the old secondary and the mature forest, is often difficult to establish.

The difference between the wet and the dry forest types is chiefly floristic and this floristic difference does not produce a fundamentally different physiognomy. The transition from one community to the other is usually gradual; one passing into the other imperceptibly except where a concomitant change of soil and climate occurs, it may then be more abrupt (Aubreville, 1938; Richard, 1952). The gradual character of this transition, but also its mosaic character (Tchouto Mbatchou, 2004) is well evidenced in our biome reconstructions in sites of southern Cameroon in the Dja forest area (Nkoul and Cyrie sites) where some sites are reconstructed as TRFO potential biome and others as TSFO potential biome, so they have all been considered as correctly reconstructed (Tables 1 and 8).

The same problem is observed at the boundary of old secondary forest and mature forest. Richards (1952) estimates that it is almost impossible to differentiate these two types of forest formation. In tropical central American forest Budowski (1970) used 3 criteria to differentiate the old secondary forest: (1) a high concentration of deciduous species in the evergreen domain, (2) abundance of lianas of low diameter and (3) the paucity in epiphytes, which are also the most characteristic criteria of the west and central old African secondary forest (Kahn, 1982). Other authors base the boundary of these two forest types in central Africa on the presence of markers such as Elaeis guineensis only in the old secondary forest (Kahn, 1982) or species of Rinorea only in the mature one (Achoundong, 1996, 2000) or in dynamic term, on the acquisition of the mechanism of regeneration by windfall that get forest species in position to regenerate in mature forest (Kahn, 1982).

But, all these criteria used by botanists in the field are very difficult to consider in our palynological work due to the lack of detailed botanical inventories at each pollen site and to the level of identification of our pollen taxa, more generally at the genus or family levels. Only the site of Kandara can illustrate this transition between old secondary forest and mature forest, pollen samples being collected along 2 contin- uous transects (Vincens et al., 2000 and unpublished data). Along these transects the boundary between the two formations has been placed following botanical inventories and mainly the presence of Rinorea species only in the mature forest (Achoundong, 2000). Unfortunately, pollen grains of Rinorea have not been identified in our samples due probably to the entomophilous character of the pollination of this plant. This boundary is not clearly identified by our pollen assemblages since some samples from the old secondary forest are reconstructed as a TMFO potential stage and some samples from the mature forest as a TOSF potential stage (Table 1). But often the calculated scores for these two stages in a same sample are very close. There is probably not a well defined boundary between these two types of forest such as between dry and wet forest; instead we suggest a transitional zone with a mosaic character. Moreover, our work being based on pollen analysis, part of pollen grains produced by the plants growing on a plot (mainly great pollen producers with well dispersed pollen grains) can be transported in a contiguous plot and so affect the local pollen rain. This could also explain why the regrowth (TRFE) and the young secondary forest (TYSF) stages are not well reconstructed by pollen assemblages, probably associated also with a high anthropogenic impact in the northern Kandara transect.

\section{Conclusions}

The application of biomisation to Atlantic Central African pollen data using a larger data set, a new and more precise classification of plant functional types and new allocations of pollen taxa to these PFTs adapted to the study area than in previous works, demonstrates that this objective and quantitative method is able to accurately predict the potential vegetation in a tropical forest region with high taxonomic diversity. In the majority of the cases, the results are comparable to site-specific descriptions of the vegetation. Savannas (SAVA potential biome), tropical rain forests (TRFO potential biome) and tropical seasonal forests (TSFO potential biome) are correctly reconstructed at $97.5 \%$ of the sites, such as the main succession stages of forest regeneration (93\% of the sites): savanna (SAVA potential stage), tropical secondary forest (TSFE potential stage) and tropical mature forest (TMFO potential stage). But inside the secondary forest the young and the pioneer stages are not clearly identified due probably to their low sampling representation. Some reconstructions can remain questionable mainly at sites located at the boundary of two forest biomes (wet rain forest and dry rain forest) or two forest stages (old secondary forest and mature forest) due to the lack of precise botanical inventories on these sites and to the fact that it does not occur a clear boundary between these forest types but a gradual and transitional zone, with also a probable mosaic character.

This work evidences the ability of modern pollen data to predict accurately the present potential vegetation in tropical 
African forest ecosystems in terms of biomes but also for the first time in Africa in terms of vegetation dynamics. These positive results open the possibility to use now with confidence fossil pollen data to reconstruct more precisely potential vegetation and its dynamics in Atlantic Central Africa during the Late Quaternary from lacustrine pollen sequences and also to refine climate past reconstructions in this region for a more accurate comparison data/modelling.

Acknowledgements. The pollen data presented in this paper were obtained in the frame of the French projects "ECOFIT" (CNRSIRD-CEA), "PRIMUS" (PNEDC-INSU-CNRS) and "REGAB" (ECLIPSE-INSU-CNRS). We thank the Institut de Recherche pour le Développement (IRD) of Pointe-Noire, Congo and of Yaoundé, Cameroon, the Cameroon Wildlife Conservation Society (CWCS), and the Institut de Recherche en Ecologie Tropicale (IRET/CENAREST, Libreville, Gabon) for their logistical help and support. We also acknowledge all the local people who assisted us during fieldworks, C. Doumenge (CIRAD, Montpellier) for constructive comments, C. Vanbesien and J. J. Motte for drawing assistance. J. Lebamba thanks the government of Gabon for the $\mathrm{PhD}$ grant 981195.

Edited by: R. Bonnefille

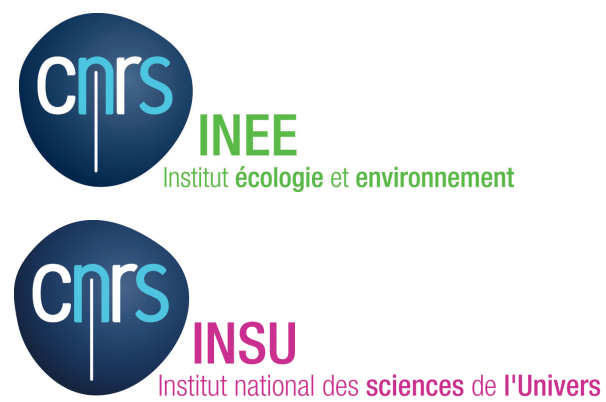

Publication of this paper was granted by EDD (Environnement, Développement Durable) and INSU (Institut des Sciences de l'Univers) at CNRS.

\section{References}

Achoundong, G.: Les Rinorea comme indicateurs des grands types forestiers du Cameroun, in: The biodiversity of African plants, edited by: van der Maesen, L. G. J., van der Burget, X. M., and van Madenbach de Rooy, J. M., Kluwer Academic Publishers, Netherlands, 536-544, 1996.

Achoundong, G.: Formation et évolution des recrûs sur savanes, in: Dynamique à long terme des écosystèmes forestiers intertropicaux, edited by: Servant, M. and Servant-Vildary, S., IRD, UNESCO, MAB, CNRS, Paris, 31-41, 2000.

African Pollen Data base: http://medias.obs-mip.fr/apd/, access: September 2008.

Aubréville, A.: La forêt coloniale: les forêts de l'Afrique occidentale française, Annales de l'Académie des Sciences coloniale, 9, $1-245,1938$.
Benzécri, J. P.: L'analyse des données, II. L'analyse des correspondances, Dunod, Paris, 1973.

Bonnefille, R., Buchet, G., Friis, I., Kelbessa, E., and Mohammed, M. U.: Modern pollen rain on an altitudinal range of forests and woodlands in South West Ethiopia, Opera Botanica, 212, 71-84, 1993.

Budowski, G.: The distinction between old secondary and climax species in tropical Central American forest, Trop. Ecol., 11, 4448, 1970.

Caballé, G.: Essai sur la géographie forestière du Gabon, Adansonia, 2, 17, 425-440, 1978.

Catinot, R., Fontaine, R. G. and Guillaumet, J. L.: Successions secondaires, in: Ecosystèmes forestiers tropicaux d'Afrique, ORSTOM - UNESCO (eds), 198-215, 1983.

Cramer, W.: Using plant functional types in a global vegetation model, in: Plant functional types, their relevance to ecosystem properties and global change, edited by: Smith, T. M., Shugart, H. H., Woodward, F. I., Cambridge University Press, Cambridge, 271-288, 1997.

De Namur, C.: Aperçu sur la végétation de l'Afrique centrale atlantique, in: Paysages quaternaires de l'Afrique centrale atlantique, edited by: Lanfranchi, R. and Schwartz, D., ORSTOM, Paris, 60-67, 1990.

De Saint Aubin, G.: Les formations végétales et composition de la forêt, in: La forêt du Gabon, Centre Technique Forestier Tropical, Nogent-sur-Marne, 13-30, 1963.

Descoings, B.: Esquisse phytogéographique du Congo, in: Atlas du Congo, ORSTOM, Paris, 1969.

Diaz, S. and Cabido, M.: Plant functional types and ecosystem function in relation to global change: an multiscale approach, J. Veg. Sci., 8, 463-474, 1997.

Diaz Barradas, M. C., Zunzunegui, M., Tirado, R., Ain-Lhout, F., and Garcia Novo, F.: Plant functional types and ecosystem function in Mediterranean shrubland, J. Veg. Sci., 10, 709-716, 1999.

Doumenge, C.: La réserve de la Conkouati : Congo. Le secteur sud-ouest, BP Exploration et UICN, Gland, Suisse, 1992.

Dowsett-Lemaire, F.: The vegetation of the Kouilou basin in Congo, Tauraco Research Report, 4, 17-51, 1991.

Elenga, H., Peyron, O., Bonnefille, R., Prentice, I. C., Jolly, D., Cheddadi, R., Guiot, J., Andrieu, V., Bottema, S., Buchet, G., de Beaulieu, J.L., Hamilton, A. C., Maley, J., Marchant, R., Perez-Obiol, R., Reille, M., Riollet, G., Scott, L., Straka, H., Taylor, D., Van Campo, E., Vincens, A., Laarif, F., and Jonson, H.: Pollen-based biome reconstruction for southern Europe and Africa 18,000 years ago, J. Biogeogr., 27, 3, 621-634, 2000a.

Elenga, H., de Namur, C., Vincens, A., Roux, M., and Schwartz, D.: Use of plots to define pollen-vegetation relationships in densely forested ecosystems of tropical Africa, Rev. Palaeobot. Palyno., 112, 1-3, 79-96, 2000b.

FAO Web LocClim: http://www.fao.org/sd/locclim/srv/en/locclim. home, access: September 2008.

Flore d'Afrique Centrale (Zaïre, Rwanda, Burundi): Jardin botanique de Belgique, Bruxelles, 1972-2004.

Flore du Cameroun : Museum National d'Histoire Naturelle, Paris, 1963-2001.

Flore du Congo Belge et du Ruanda-Urundi: Publications de l'Institut National pour l'Etude Agronomique du Congo Belge, Bruxelles, 1948-1963.

Flore du Congo, du Rwanda et du Burundi: Jardin Botanique Na- 
tional de Belgique, Bruxelles, 1967-1971.

Flore du Gabon: Museum National d'Histoire Naturelle, Paris, 1961-2004.

Giresse, P., Makaya Mvoubou, Maley, J. and Ngomanda, A.: LateHolocene equatorial environments inferred from deposition processes, carbon isotopes of organic matter, and pollen in three shallow lakes of Gabon, west central Africa, J. Paleolimnol., 41, 2, 369-392,, 2008.

Gitay, H. and Noble, I. R.: What are functional types and how should we seek them?, in: Plant functional types, their relevance to ecosystem properties and global change, edited by: Smith, T. M., Shugart, H. H., and Woodward, F. I., Cambridge University Press, Cambridge, 3-19, 1997.

Haxeltine, A. and Prentice, I. C.: BIOME3: an equilibrium terrestrial biosphere model based on ecophysiological constraints, resource availability, and competition among plant functional type, Global Biogeochem. Cy., 10, 693-709, 1996.

Hecketsweiler, P. and Mokoko Ikonga, J.: La réserve de la Conkouati: Congo. Le secteur sud-est., BP Exploration and UICN, Gland, Suisse, 4, 323 pp., 1991.

Hély, C., Bremond, L., Alleaume, S., Smith, B., Sykes, M., and Guiot, J.: Sensitivity of African biomes to changes in the precipitation regime, Global Ecol. Biogeogr., 15, 258-270, 2006.

Hutchinson, J. and Dalziel, J. M.: Flora of West Tropical Africa, Whitefriars, London, 1954-1972.

Jolly, D., Bonnefille, R., Burcq, S., and Roux, M.: Représentation pollinique de la forêt dense humide du Gabon, tests statistiques, C. R. Acad. Sci., Paris, 322, 1, 63-70, 1996.

Jolly, D. and Haxeltine, A.: Effect of low glacial atmospheric CO2 on tropical African montane vegetation, Science, 276, 786-788, 1997.

Jolly, D., Harrison, S. P., Damnati, B., and Bonnefille, R.: Simulated climate and biomes of Africa during the Late Quaternary: comparison with pollen and lake status data, Quaternary Sci. Rev., 17, 629-657, 1998a.

Jolly, D., Prentice, I. C., Bonnefille, R., Ballouche, A., Bengo, M., Brenac, P., Buchet, G., Burney, D., Cazet, J. P., Cheddadi, R., Edorh, T., Elmoutaki, S., Guiot, J., Laarif, F., Lamb, H., Lezine, A. M., Maley, J., Mbenza, M., Peyron, O., Reille, M., Reynaud-Farrera, I., Riollet, G., Ritchie, J. C., Roche, E., Scott, L., Ssemmanda, I., Straka, H., Umer, M., Van Campo, E., Vilimumbalo, S., Vincens, A., and Waller, M.: Biome reconstruction from pollen and plant macrofossil data for Africa and the Arabian peninsula at 0 and 6000 years, J. Biogeogr., 25, 1007-1027, 1998b.

Kahn, F.: La reconstitution de la forêt tropicale humide. Sud-Ouest de la Côte d'Ivoire, ORSTOM, Paris, mémoire 97, 1982.

Köhler, P., Ditzer, T., and Huth, A.: Concepts fort he aggregation of tropical tree species into functional types and the application to Sabah's lowland rain forests, J. Trop. Ecol., 16, 591-602, 2000.

Lavorel, S., McIntyre, S., Landsberg, J., and Forbes, D.: Plant functional classifications: from general groups to specific groups based on response to disturbance, Trends Ecol. Evol., 12, 474478, 1997

Lavorel, S. and Garnier, E.: Predicting the effects of environmental changes on plant community composition and ecosystem functioning: revisiting the Holy Grail, Funct. Ecol., 16, 545-556, 2002

Lavorel, S., Diaz, S., Cornelissen, J. H. C., Garnier, E., Harrison, S.,
McIntyre, S., Pausas Natalia Pérez-Harguindeguy, J. G., Roumet, C., and Urcelay, C.: Plant functional types: are we getting any closer to the Holy Grail ?, in: Terrestrial ecosystems in a changing world, Canadell, J. G., Pataki, D. and Pitelka, L., The IGBP Series, Springer-Verlag, Berlin Heidelberg, 149-164, 2007.

Lebamba, J., Vincens A., Jolly, D., Ngomanda, A., Schevin, P., Maley, J., Bentaleb, I., and REGAB Members: Modern pollen rain in savanna and forest ecosystems of Gabon and Cameroon, Atlantic Central Africa, Rev. Palaeobot. Palyno., 153, 34-45, 2009.

Lebrun, J.-P. and Stork, A. L.: Enumération des plantes à fleurs d'Afrique tropicale, Conservatoire et Jardins Botaniques, Genève, 1991-1997.

Lebrun, J.-P. and Stork, A. L.: Tropical African flowering plants, Ecology and distribution, Vol. 1: Annonaceae-Balanitaceae, Conservatoire et Jardin Botaniques, Genève, 2003.

Lebrun, J.-P. and Stork, A. L.: Tropical African flowering plants, Ecology and distribution, Vol. 2: EuphorbiaceaeDichapetalaceae, Conservatoire et Jardin Botaniques, Genève, 2006.

Leemans, R.: The use of plant functional type classifications to model global land cover and simulate the interactions between the terrestrial biosphere and the atmosphere, in: Plant functional types, their relevance to ecosystem properties and global change, edited by: Smith, T. M., Shugart, H. H., and Woodward, F. I., Cambridge University Press, Cambridge, 289-317, 1997.

Letouzey, R.: La forêt à Lophira alata de la zone littorale camerounaise, Bois et Forêts des Tropiques, 53, 9-20, 1957.

Letouzey, R.: Etude phytogéographique du Cameroun, Lechevalier, Paris, 1968.

Letouzey, R.: Notice de la carte phytogéographique du Cameroun au 1: 500 000. Institut de la Carte Internationale de la Végétation, Toulouse, France, 1985.

Lézine, A.-M. and Edorh, T.: Modern pollen deposition in West African Sudanian environments, Rev. Palaeobot. Palyno., 67, 4158, 1991.

Lézine, A.-M., Watrin, J., Vincens, A., Hély, C., and Contributors: Are modern pollen data representative of the west African vegetation?, Rev. Palaeobot. Palyno., doi: 10.1016/j.revpalbo.2009.02.001, 2009.

Maley, J.: L'histoire récente de la forêt dense humide africaine : essai sur la dynamique de quelque formation forestière, in: Paysages quaternaires de l'Afrique centrale atlantique, edited by: Lanfranchi, R. and Schwartz, D., ORSTOM, Paris, 367-382, 1990.

Maley, J. and Elenga, H.: Le rôle des nuages dans l'évolution des paléoenvironnements montagnards de l'Afrique tropicale, Veille Climatique Satellitaire, 46, 51-63, 1993.

Mayaux, P., Janodet, E., Blair-Myers, C., and Legeay-Janvier, P.: Vegetation map of central Africa at 1:5000 000. Tropical Ecosystem Environment Observation by Satellites, TREES series D, 1, 1997.

Mayaux, P., Richards, T., and Janodet, E.: A vegetation map of Central Africa derived from satellite imagery, J. Biogeogr, 25, 353-366, 1999.

Moutsamboté, J. M., N'zala, D., and Ngondo, J. C.: Evolution des recrus forestiers après culture de manioc au Mayombe (Congo), Cahiers d'Etudes et de Recherches Francophones, Agriculture, 9, 2, 141-144, 2000.

Ngomanda, A.: Dynamique des écosystèmes forestiers du Gabon 
au cours des cinq derniers millénaires, Ph.D. thesis, University of Montpellier 2, France, 2005.

Nicholson, S. E.: The nature of rainfall variability over Africa on time scales of decades to millennia, Global Planet. Change, 26, 137-158, 2000

Nicolas, P.: Contribution à l'étude phytogéographique de la forêt du Gabon, Ph.D. thesis, University Paris I, France, 1977.

Noble, I. R. and Gitay, H. A.: A functional classification for predicting the dynamics of landscapes, J. Veg. Sci., 7, 329-336, 1996.

Peyron, O., Jolly, D., Bonnefille, R., Vincens, A., and Guiot, J.: Climate of east Africa $6000{ }^{14} \mathrm{C}$ Yr B.P. as inferred from pollen data, Quaternary Res., 54, 90-101, 2000.

Peyron, O., Jolly, D., Braconnot, P., Bonnefille, R., Guiot, J., Wirrmann, D., and Chalié, F.: Quantitative reconstructions of annual rainfall in Africa 6000 years ago: model-data comparison, J. Geophys. Res., 111, D24110, doi:10.1029/2006.JD007396, 2006

Prentice, I. C., Cramer, W., Harrison, S. P., Leemans, R., Monserud, R. A., and Solomon, A. M.: A global biome model based on plant physiology and dominance, soil properties and climate, J. Biogeogr., 19, 117-134, 1992.

Prentice, I. C., Guiot, J., Huntley, B., Jolly, D. and Cheddadi, R.: Reconstructing biomes from palaeoecological data: a general method and its application to European pollen data at 0 and $6 \mathrm{ka}$, Clim. Dynam., 12, 185-194, 1996.

Prentice, I. C. and Webb III, T.: Biome 6000: reconstructing global mid-Holocene vegetation patterns from palaeoecological records, J. Biogeogr., 25, 997-1053, 1998.

Prentice, I. C., Jolly, D. and BIOME 6000 participants: MidHolocene and glacial-maximum vegetation geography of the northern continents and Africa, J. Biogeogr., 27, 507-519, 2000.

Richards, P. W.: The tropical rain forest. An ecological study, Cambridge University Press, Cambridge, 450 pp., 1952.

Robyns, W.: Contribution à l'étude des formations herbeuses du district forestier central du Congo Belge, Mém, Inst. Roy. Colon. Belge, 5, 1936.

Schnell, R.: Introduction à la phytogéographie des pays tropicaux. 3. La flore et la végétation de l'Afrique tropicale, GauthierVillars, Paris, 1976.

Schwartz, D., Elenga, H., Vincens, A., Bertaux, J., Mariotti, A., Achoundong, G., Alexandre, A., Belingard, C., Girardin, C., Guillet, B., Maley, J., de Namur, C., Reynaud-Farrera, I., and Youta Happi, J.: Origine et évolution des savanes des marges forestières en Afrique centrale Atlantique (Cameroun, Gabon, Congo): approche aux échelles millénaires et séculaires, in: Dynamique à long terme des écosystèmes forestiers intertropicaux, edited by: Servant, M. and Servant-Vildary, S., IRD, UNESCO, MAB, CNRS, Paris, 325-338, 2000.
Sita, P.: La forêt tropicale au Congo, in : Hommes et environnement, Quarante ans de recherche scientifique au Congo, Conférences de l'ORSTOM, Brazzaville novembre 1989, ORSTOM, 104-110, 1989.

Skarpe, C.: Plant functional types and climate in a southern African savanna, J. Veg. Sci., 7, 397-404, 1996.

Smith, T. M., Shugart, H. H., and Woodward, F. I.: Plant functional types, their relevance to ecosystem properties and global change, Cambridge University Press, Cambridge, 1997.

Tchouto Mbatchou, G. P.: Plant diversity in a Central African forest. Implications for biodiversity conservation in Cameroon, Tropenbos International, Publications, Cameroon series 7, 210 pp., 2004.

Vincens, A., Ssemmanda, I., Roux, M., and Jolly, D.: Study of the modern pollen rain in western Uganda with a numerical approach, Rev. Palaeobot. Palyno., 96, 145-168, 1997.

Vincens, A., Dubois, M. A., Guillet, B., Achoundong, G., Buchet, G., Kamgang Kabeyene Beyala, V., de Namur, C., and Riéra, B.: Pollen-rain-vegetation relationships along a forest-savanna transect in southeastern Cameroon, Rev. Palaeobota. Palyno., 110, 3-4, 191-208, 2000.

Vincens, A., Bremond, L., Brewer, S., Buchet, G., and Dussouillez, P.: Modern pollen-based biome reconstructions in East Africa expanded to southern Tanzania, Rev. Palaeobot. Palyno., 140, 187-212, 2006.

Vincens, A., Lézine, A.-M., Buchet, G., Lewden, D., Le Thomas, A., and contributors: African Pollen Database inventory of tree and shrub pollen types, Rev. Palaeobot. Palyno., 145, 135-141, 2007.

Ward, J. H.: Hierarchical grouping to optimize an objective function, J. Am. Stat. Assoc., 58, 236-244, 1963.

White, F.: The vegetation of Africa. A descriptive memoir to accompany the UNESCO/AETFAT/UNSO vegetation map of Africa, UNESCO, Paris, 1983.

White, L. J. T. and Abernethy, K.: Le guide de la végétation de la Réserve de La Lopé, ECOFAC-GABON, Libreville, 1996.

Woodward, F. I. and Cramer, W.: Plant functional types and climatic changes: introduction, J. Vege. Sci., 7, 306-308, 1996.

Wright, H. E.: The use of surface samples in Quaternary pollen analysis, Rev. Palaeobot. Palyno., 2, 321-330, 1967. 